\title{
ON THE CORE AND WALRASIAN EXPECTATIONS EQUILIBRIUM IN INFINITE DIMENSIONAL COMMODITY SPACES
}

\author{
ANUJ BHOWMIK AND JILING CAO
}

\begin{abstract}
In this paper, we establish two different characterizations of Walrasian expectations allocations by the veto power of the grand coalition in an asymmetric information economy having finite numbers of agents and states of nature and whose commodity space is a Banach lattice. The first one deals with Aubin non-dominated allocations, and the other claims that an allocation is a Walrasian expectations allocation if and only if it is not privately dominated by the grand coalition, by considering perturbations of the original initial endowments in precise directions.
\end{abstract}

\section{INTRODUCTION}

The classical deterministic Arrow-Debreu-McKenzie model on an economic system consists of finitely many consumers, producers and commodities, refer to 3 and [17. In late of 1950's, Arrow and Debreu introduced uncertainty into this deterministic model by adding contingent claims, [6, Chapter 7]. In this improved model, agents make contracts contingent on the realized state of nature known to all the agents. However, such a model does not capture the idea of contracts under asymmetric information as all agents face the same uncertainty. To overcome this shortcoming, Radner 21] introduced economies with asymmetric information. In Radner's model, an economy consists of finitely many agents, each of whom is characterized by a state dependent utility function, a random initial endowment, a private information set and his prior belief; and agents arrange contingent contracts for trading commodities before they obtain any information about the realized state of nature. Analogous to the concept of a Walrasian equilibrium in the Arrow-Debreu-McKenzie model, Radner also introduced the notion of a Walrasian expectations equilibrium for an asymmetric information economy so that the information of an agent places a restriction on his feasible trades: better information allows for more contingent trades, and each agent maximizes his ex ante expected utility subject to his budget constraint with respect to his private information. This individualistic behavior leads to a feasible redistribution of the initial endowments for each state of nature.

In this paper, we continue the study on asymmetric information economies. In particular, we are interested in asymmetric information economies whose commodity spaces are infinite dimensional spaces. In Section 2, we give a general description on a discrete model of an asymmetric information economy having finitely many of

JEL Classification Numbers: D51, D82, D11.

Keywords. Asymmetric information economy; Aubin non-dominated allocation; Private core; Privately non-dominated allocation; Properness; Walrasian expectations allocation. 
agents and states of nature, and whose commodity space is a Banach lattice. We also associate a continuum model with this discrete model. The continuum model has the equal treatment property. It is worth to mention that Tourky and Yannelis 24] and Podczeck [19] constructed counterexamples of economies to show that the classical core-Walras equivalence in [5] fails whenever the commodity space is a non-separable ordered Banach space. In both of these two papers, the authors used the Continuum Hypothesis in set theory to construct an economy with uncountably many utility functions. Since the commodity spaces of economies in our paper are Banach lattices which are not necessarily separable, our theorems give some positive results for asymmetric information economies whose commodity spaces are non-separable.

The concept of the private core of an asymmetric information economy was introduced in [27]. Einy et al. [8] showed that under appropriate assumptions, the private core coincides with the set of Walrasian expectations allocations for an atomless economy whose commodity space is Euclidean space. Hervés-Beloso et al. [15] established a similar result for an equal treatment economy whose commodity space is $\ell_{\infty}$. By considering an atomless economy with asymmetric information, Evren and Hüsseinov 9] extended these results to an economy whose commodity space is an ordered separable Banach space which has an interior point in its positive cone. In Section 3, we establish a similar equivalence result for an equal treatment economy whose commodity space is a Banach lattice. In an atomless economy with a complete information and a finite dimensional commodity space, improving a result of [5], Schmeidler [23] and Grodal [12] showed that (i) if some coalition blocks an allocation, then there is also a blocking subcoalition with arbitrarily small measure; and (ii) the small coalition can be the union of at most $\ell+1$ coalitions, each of which has measure and diameter less than an arbitrary small number $\epsilon>0$, where $\ell$ is the number of commodities. In the same issue of Econometrica, Vind [26] extended (i) to show that if some coalition blocks then there is a blocking coalition with any measure less than the measure of the grand coalition. The first extension of the Schmeidler and Grodal's results to an infinite dimensional setting (the space $\ell_{\infty}$ ) were obtained in [13, where it was also showed that the Vind's result fails in the space $\ell_{\infty}$ under the standard assumptions. Hervés-Beloso et al. [14] first extended Vind's theorem to an asymmetric information economy with a continuum of agents having the equal treatment property, and whose commodity space is Euclidean space. Later, Hervés-Beloso et al. [15] extended Vind's theorem to an asymmetric information economy with a continuum of agents having the equal treatment property and whose commodity space is $\ell_{\infty}$. All of these extension are established by using finite-dimensional Lyapunov's convexity theorem. Using an infinite dimensional extension of Lyapunov's convexity theorem, Evren and Hüsseinov [9] further extended Vind's theorem to an atomless economy whose commodity space is an ordered Banach space having an interior point in its positive cone, with some additional assumption. Our second main result in Section 3 is an extension of Vind's theorem to an asymmetric information economy with the equal treatment property, and whose commodity space is a Banach lattice.

Addressing complete information economies with finitely many agents and commodities, Aubin 4 introduced the ponder veto concept and showed that the core obtained by this veto mechanism coincides with the Walrasian equilibria. Aubin's approach was employed by Gabriella Graziano, Meo and Hervés-Beloso et al. to 
characterize Walrasian expectations equilibria of asymmetric information economies. Hervés-Beloso et al. 14] introduced the notion of Aubin non-dominated allocations. Gabriella Graziano and Meo [10] showed that the Aubin private core provides a complete characterization of Walrasian expectations allocations in an economy with a complete measure space of agents. They also showed that Aubin private core allocations in an asymmetric information economy with a complete measure space of agents and an ordered separable Banach space whose positive cone has an interior point as the commodity space can be characterized by privately non-dominated allocations in suitable associated economies. Hervés-Beloso et al. 14, 15] established similar results for asymmetric information economies with finitely many agents and states of nature, and $\mathbb{R}^{\ell}$ or $\ell_{\infty}$ as the commodity space. The proof of Hervés-Beloso et al. was built on associated continuum economies and thus requires the validity of Vind's theorem, which is not the case in the proof of Gabriella Graziano and Meo [10. In Section 4, we adopt the approach in [14, 15] to characterize Walrasian expectations equilibria in terms of the private blocking power of the grand coalition. Since our economic model has finitely many agents and a Banach lattice as the commodity space, our results can be considered as extensions of those in [14, 15].

In the Appendix, an asymmetric information economy whose commodity space is a Banach lattice and which has infinitely many states of nature is discussed. Mathematical preliminaries and discussions on some assumptions are also provided in the Appendix. Of course, all of these mathematical preliminaries can be found in [1].

\section{Description OF THE MODEL}

In this section, we describe our model of an exchange economy with asymmetric information.

2.1. The discrete model. Our first model of an exchange economy is a discrete model $\mathcal{E}$ with (fixed) $n$ agents denoted by the set $N=\{1, \ldots, n\}$, like those ones considered in $[21,22$. A measurable space $(\Omega, \mathcal{F})$ is used to describe the exogenous uncertainty of $\mathcal{E}$, where $\Omega$ is a finite set denoting the set of all possible states of nature and the $\sigma$-algebra $\mathcal{F}$ denotes the set of all events. The commodity space of $\mathcal{E}$ is a Banach lattice $Y$ with a partial order $\leq$. The economy $\mathcal{E}$ extends over two time periods $\tau=0,1$, and consumption takes place at $\tau=1$. At $\tau=0$, there is uncertainty over the states of nature and agents make contracts that may be contingent on the realized state of nature at $\tau=1$. More precisely, $\mathcal{E}$ is expressed by $\mathcal{E}=\left\{\left((\Omega, \mathcal{F}), Y_{+}, \mathcal{F}_{i}, U_{i}, a_{i}, q_{i}\right): i \in N\right\}$, where the positive cone $Y_{+}$of $Y$ is the consumption set in each state of nature $\omega \in \Omega$ for every agent $i \in N ; \mathcal{F}_{i}$ is a partition of $\Omega$ representing the private information of agent $i ; U_{i}: \Omega \times Y_{+} \rightarrow \mathbb{R}$ is the random utility function of agent $i ; a_{i}: \Omega \rightarrow Y_{+}$is the random initial endowment of agent $i$, assumed to be constant on elements of $\mathcal{F}_{i}$; and $q_{i}$ is a probability measure on $\Omega$ giving the prior of agent $i$, assumed to be positive on all elements of $\Omega$. For any random consumption bundle $x: \Omega \rightarrow Y_{+}$, the ex ante expected utility of agent $i$ is given by $V_{i}(x)=\sum_{\omega \in \Omega} U_{i}(\omega, x(\omega)) q_{i}(\omega)$.

An assignment in $\mathcal{E}$ is a function $x=\left(x_{1}, \ldots, x_{n}\right)$ which associates to every agent $i$ a random consumption bundle $x_{i}: \Omega \rightarrow Y_{+}$, equivalently written as $x_{i} \in\left(Y_{+}\right)^{\Omega}$. We call a function with domain $\Omega$, constant on elements of $\mathcal{F}_{i}, \mathcal{F}_{i}$-measurable, although measurability is meant with respect to the $\sigma$-algebra generated by the partition. Put $L_{i}=\left\{x_{i} \in\left(Y_{+}\right)^{\Omega}: x_{i}\right.$ is $\mathcal{F}_{i}$-measurable $\}$. An assignment $x=\left(x_{1}, \ldots, x_{n}\right)$ in 
$\mathcal{E}$ is called an allocation if $x_{i} \in L_{i}$ for all $i \in N$. An allocation $x$ is said to be feasible if $\sum_{i=1}^{n} x_{i}(\omega) \leq \sum_{i=1}^{n} a_{i}(\omega)$ for all $\omega \in \Omega$. Further, an allocation $x$ of $\mathcal{E}$ is privately dominated if there exists a feasible allocation $y=\left(y_{1}, \ldots, y_{n}\right)$ such that $V_{i}\left(y_{i}\right)>V_{i}\left(x_{i}\right)$ for all $i \in N$. A feasible allocation $x$ of $\mathcal{E}$ is called privately Pareto optimal if it is not privately dominated. A price system is an $\mathcal{F}$-measurable, nonzero function $\pi: \Omega \rightarrow Y_{+}^{*}$, where $Y_{+}^{*}$ is the positive cone of the norm-dual space $Y^{*}$ of $Y$. The budget set $B_{i}(\pi)$ of agent $i$ under $\pi$ is defined as

$$
B_{i}(\pi)=\left\{x_{i} \in L_{i}: \sum_{\omega \in \Omega}\left\langle\pi(\omega), x_{i}(\omega)\right\rangle \leq \sum_{\omega \in \Omega}\left\langle\pi(\omega), a_{i}(\omega)\right\rangle\right\} .
$$

Given a feasible allocation $x$ and a price system $\pi$ in $\mathcal{E}$, the pair $(x, \pi)$ is called a Walrasian expectations quasi-equilibrium of $\mathcal{E}$ in the sense of Radner if

(2.1) for all $i \in N, x_{i} \in B_{i}(\pi)$ and the consumption bundle $x_{i}$ maximizes $V_{i}$ on $B_{i}(\pi)$ whenever $\sum_{\omega \in \Omega}\left\langle\pi(\omega), a_{i}(\omega)\right\rangle \neq 0$

(2.2) $\sum_{\omega \in \Omega}\left\langle\pi(\omega), \sum_{i \in N} x_{i}(\omega)\right\rangle=\sum_{\omega \in \Omega}\left\langle\pi(\omega), \sum_{i \in N} a_{i}(\omega)\right\rangle$.

If $\sum_{\omega \in \Omega}\left\langle\pi(\omega), a_{i}(\omega)\right\rangle \neq 0$ for some $i \in N$, then $(x, \pi)$ is called non-trivial. Further, if

$\left(2.1^{\prime}\right)$ for all $i \in N, x_{i} \in B_{i}(\pi)$ and $x_{i}$ maximizes $V_{i}$ on $B_{i}(\pi)$,

and $(2.2)$ hold, $(x, \pi)$ is called a Walrasian expectations equilibrium of $\mathcal{E}$ in the sense of Radner, and $x$ is called a Walrasian expectations allocation. Note that if $(x, \pi)$ is a Walrasian expectations quasi-equilibrium, then feasibility of $x$ and $(2.2)$ together imply

(2.3) $\left\langle\pi(\omega), \sum_{i \in N} x_{i}(\omega)\right\rangle=\left\langle\pi(\omega), \sum_{i \in N} a_{i}(\omega)\right\rangle$ for each $\omega \in \Omega$.

Throughout this paper, we put the following assumptions on our discrete model $\mathcal{E}$. These assumptions or some of their combinations are used in different places of the paper.

$\left(\mathrm{A}_{1}\right)$ Continuity. For each $i \in N$ and $\omega \in \Omega, U_{i}(\omega, \cdot)$ is norm-continuous.

$\left(\mathrm{A}_{2}\right)$ Monotonicity. For each $i \in N$ and $\omega \in \Omega, U_{i}(\omega, \cdot)$ is monotone in the sense that if $x, y \in Y_{+}$with $y>0$, then $U_{i}(\omega, x+y)>U_{i}(\omega, x)$.

$\left(\mathrm{A}_{3}\right)$ Concavity. For each $i \in N$ and $\omega \in \Omega, U_{i}(\omega, \cdot)$ is concave.

$\left(\mathrm{A}_{4}\right)$ Quasi-interiority. For each $\omega \in \Omega, \sum_{i \in N} a_{i}(\omega) \gg 0$.

$\left(\mathrm{A}_{4}^{\prime}\right)$ Positivity. For each $\omega \in \Omega, \sum_{i \in N} a_{i}(\omega)>0$.

$\left(\mathrm{A}_{5}\right)$ Strong positivity. For each $i \in N, \inf \left\{a_{i}(\omega): \omega \in \Omega\right\}>0$.

$\left(\mathrm{A}_{6}\right)$ Stability. There exists an element $\hat{a} \in Y_{+}$such that $L\left(\sum_{i \in N} a_{i}(\omega)\right)=L(\hat{a})$ for each $\omega \in \Omega$.

$\left(\mathrm{A}_{7}\right)$ Irreducibility. For each feasible allocation $x$ of $\mathcal{E}$ and any two non-empty disjoint subsets $N_{1}, N_{2}$ of $N$ with $N_{1} \cup N_{2}=N$, there is a $y=\left(y_{i}\right)_{i \in N_{2}}$ such that $y_{i} \in L_{i}$ and $V_{i}\left(y_{i}\right)>V_{i}\left(x_{i}\right)$ for all $i \in N_{2}$, and $\sum_{i \in N_{1}} a_{i}(\omega)+$ $\sum_{i \in N_{2}} x_{i}(\omega) \geq \sum_{i \in N_{2}} y_{i}(\omega)$ for each $\omega \in \Omega$.

Remark 2.1. Under $\left(\mathrm{A}_{1}\right), V_{i}$ is continuous with respect to the product topology induced by the norm. Under $\left(\mathrm{A}_{2}\right), V_{i}$ is monotone in the sense that if $x, y \in\left(Y_{+}\right)^{\Omega}$ with $y(\omega)>0$ for some $\omega \in \Omega$, then $V_{i}(x+y)>V_{i}(x)$. Under $\left(\mathrm{A}_{3}\right), V_{i}$ is concave. $\left(\mathrm{A}_{6}\right)$ implies that the aggregate endowments do not vary too much across states. $\left(\mathrm{A}_{7}\right)$ is similar to $(A .3)$ in $[8]$. When $Y=\ell_{\infty}$, assumptions $\left(\mathrm{A}_{2}\right)-\left(\mathrm{A}_{4}\right)$ are similar to (A.2)-(A.4) in [15]. Further, assumptions $\left(\mathrm{A}_{1}\right)-\left(\mathrm{A}_{4}\right)$ are similar to (A.2) and (A.3) in [10, and (A.A3)-(A.A5) in [9] (except for the convexity). 
The following proposition, which is a modification of Proposition 3.2 in [8], shall be used in the sequel.

Proposition 2.2. If $\mathcal{E}$ satisfies $\left(\mathrm{A}_{7}\right)$, then every non-trivial Walrasian expectations quasi-equilibrium of $\mathcal{E}$ is a Walrasian expectations equilibrium.

Proof. Let $(x, \pi)$ be a non-trivial Walrasian expectations quasi-equilibrium of $\mathcal{E}$. Then $N_{2}=\left\{i \in N: \sum_{\omega \in \Omega}\left\langle\pi(\omega), a_{i}(\omega)\right\rangle \neq 0\right\} \neq \emptyset$. Let $N_{1}=N-N_{2}$. If $N_{2}=N$, there is nothing to verify. Otherwise, $N_{1} \neq \emptyset$. By $\left(\mathrm{A}_{7}\right)$, there is a $y=\left(y_{i}\right)_{i \in N_{2}}$ such that $y_{i} \in L_{i}$ and $V_{i}\left(y_{i}\right)>V_{i}\left(x_{i}\right)$ for all $i \in N_{2}$, and $\sum_{i \in N_{1}} a_{i}(\omega)+$ $\sum_{i \in N_{2}} x_{i}(\omega) \geq \sum_{i \in N_{2}} y_{i}(\omega)$ for all $\omega \in \Omega$. Then $\sum_{i \in N_{2}} \sum_{\omega \in \Omega}\left\langle\pi(\omega), x_{i}(\omega)\right\rangle \geq$ $\sum_{i \in N_{2}} \sum_{\omega \in \Omega}\left\langle\pi(\omega), y_{i}(\omega)\right\rangle$, and $\sum_{\omega \in \Omega}\left\langle\pi(\omega), x_{i}(\omega)\right\rangle<\sum_{\omega \in \Omega}\left\langle\pi(\omega), y_{i}(\omega)\right\rangle$ for each $i \in N_{2}$, which is a contradiction.

2.2. A continuum interpretation. Now, we associate a continuum economy $\mathcal{E}_{c}$ with the discrete model $\mathcal{E}$, just like that in [1] and [14, 15]. The set of agents of $\mathcal{E}_{c}$ is the unit interval $I=[0,1]$ endowed with the Lebesgue measure $\mu$. We write $I=\bigcup_{i=1}^{n} I_{i}$, where $I_{i}=\left[\frac{i-1}{n}, \frac{i}{n}\right)$ for $i=1, \ldots, n-1$, and $I_{n}=\left[\frac{n-1}{n}, 1\right]$. Each agent $t \in I_{i}$ is characterized by the private information set $\mathcal{F}_{t}=\mathcal{F}_{i}$; the consumption set $Y_{+}$in each state $\omega \in \Omega$; the random initial endowment $a(t, \cdot)=a_{i}$; the random utility function $U_{t}=U_{i}$; and the prior $q_{t}=q_{i}$. The ex ante expected utility function of every agent $t \in I_{i}$ is $V_{t}=V_{i}$. Thus, $\mathcal{E}_{c}$ can be expressed as $\mathcal{E}_{c}=$ $\left\{\left((\Omega, \mathcal{F}), Y_{+}, I, \mathcal{F}_{i}, a_{i}, V_{i}, q_{i}\right): i \in N\right\}$. We call $I_{i}$ the set of type $i$ agents, and $\mathcal{E}_{c}$ an economy with the equal treatment property. An assignment in $\mathcal{E}_{c}$ is a function $f: I \times \Omega \rightarrow Y_{+}$such that $f(\cdot, \omega) \in L_{1}\left(\mu, Y_{+}\right)$for all $\omega \in \Omega$, where $L_{1}\left(\mu, Y_{+}\right)$is the set of all Bochner integrable functions from $I$ into $Y_{+}$. Put $L_{t}=L_{i}$ for each $t \in I_{i}$ and $i \in N$. An assignment $f$ in $\mathcal{E}_{c}$ is called an allocation if $f(t, \cdot) \in L_{t}$ for almost all $t \in I$. An allocation $f$ in $\mathcal{E}_{c}$ is feasible if $\int_{I} f(t, \omega) d \mu(t) \leq \int_{I} a(t, \omega) d \mu(t)$ for all $\omega \in \Omega$. A coalition in $\mathcal{E}_{c}$ is a Borel measurable subset $S \subseteq I$ with $\mu(S)>0$. A coalition $S$ privately blocks an allocation $f$ in $\mathcal{E}_{c}$ if there is a function $g: S \times \Omega \rightarrow Y_{+}$ such that $g(t, \cdot) \in L_{t}$ and $V_{t}(g(t, \cdot))>V_{t}(f(t, \cdot))$ for all $t \in S$, and $\int_{S} g(t, \omega) d \mu(t) \leq$ $\int_{S} a(t, \omega) d \mu(t)$ for all $\omega \in \Omega$. The private core of $\mathcal{E}_{c}$ is the set of all feasible allocations which are not privately blocked by any coalition.

Given a feasible allocation $f$ and a price system $\pi$ in $\mathcal{E}_{c}$, the budget set of an agent $t \in I$ is $B_{t}(\pi)=B_{i}(\pi)$ if $t \in I_{i}$ and $i \in N$. The pair $(f, \pi)$ is called a Walrasian expectations quasi-equilibrium of $\mathcal{E}_{c}$ in the sense of Radner if

(2.4) for all $t \in I, f(t, \cdot) \in B_{t}(\pi)$ and $f(t, \cdot)$ maximizes $V_{t}$ on $B_{t}(\pi)$ whenever $\sum_{\omega \in \Omega}\langle\pi(\omega), a(t, \omega)\rangle \neq 0$

(2.5) $\sum_{\omega \in \Omega}\left\langle\pi(\omega), \int_{I} f(t, \omega) d \mu(t)\right\rangle=\sum_{\omega \in \Omega}\left\langle\pi(\omega), \int_{I} a(t, \omega) d \mu(t)\right\rangle$.

If $\sum_{\omega \in \Omega}\langle\pi(\omega), a(t, \omega)\rangle \neq 0$ for all $t$ in some coalition $S \subseteq I$, then $(f, \pi)$ is called non-trivial. Further, if

$\left(2.4^{\prime}\right)$ for all $t \in I, f(t, \cdot) \in B_{t}(\pi)$ and $f(t, \cdot)$ maximizes $V_{t}$ on $B_{t}(\pi)$

and (2.5) hold, then $(f, \pi)$ is called a Walrasian expectations equilibrium of $\mathcal{E}_{c}$ in the sense of Radner, and $f$ is called a Walrasian expectations allocation. An allocation $f$ in $\mathcal{E}_{c}$ can be interpreted as an allocation $x$ in $\mathcal{E}$, where $x_{i}=n \int_{I_{i}} f(t, \cdot) d \mu(t)$ for all $i \in N$. Conversely, an allocation $x$ in $\mathcal{E}$ can be interpreted as an allocation $f$ in $\mathcal{E}_{c}$, where $f$ is the simple function given by $f(t, \cdot)=x_{i}$ for all $t \in I_{i}$ and $i \in N$. 
Analogous to Theorem 1 in [11] and Theorem 3.1 in [14, 15, our next result shows that the discrete and continuum approaches can be considered equivalent with respect to Walrasian expectations (quasi-)equilibria.

Proposition 2.3. Assume that $\mathcal{E}$ satisfies $\left(\mathrm{A}_{3}\right)$. If $(x, \pi)$ is a non-trivial Walrasian expectations quasi-equilibrium of $\mathcal{E}$, then $(f, \pi)$ is a non-trivial Walrasian expectations quasi-equilibrium of $\mathcal{E}_{c}$, where $f(t, \cdot)=x_{i}$ if $t \in I_{i}$. Conversely, if $(f, \pi)$ is a non-trivial Walrasian expectations quasi-equilibrium of $\mathcal{E}_{c}$, then $(x, \pi)$ is a nontrivial Walrasian expectations quasi-equilibrium of $\mathcal{E}$, where $x_{i}=n \int_{I_{i}} f(t, \cdot) d \mu(t)$.

Since the proof of Proposition 2.3 is straightforward, we omit it.

Remark 2.4. A similar conclusion holds if "non-trivial Walrasian expectations quasi-equilibrium" is replaced with "Walrasian expectations equilibrium".

\section{Characterizations of private cores of EQUal treatment economies}

In this section, we establish a relation between the private core and the set of Walrasian expectations allocations in the setting of equal treatment, and give an extension of Vind's theorem. These two results allow us to obtain our main theorems in Section 4.4 .

3.1. Equivalence results. Evren and Hüsseinov [9] provided an equivalence theorem between the private core and the set of Walrasian expectations allocations in an economy whose commodity space is an ordered separable Banach space having an interior point in its positive cone. Next, we give a similar result for the case that the commodity space is a Banach lattice.

Theorem 3.1. Assume that the commodity space of $\mathcal{E}$ has an interior point in its positive cone. Let $f$ be a feasible allocation in $\mathcal{E}_{c}$ such that $f(t, \cdot)=x_{i}$ for all $t \in I_{i}$ and $i \in N$. Under $\left(\mathrm{A}_{1}\right),\left(\mathrm{A}_{2}\right)$ and $\left(\mathrm{A}_{4}\right)$, if $f$ is in the private core of $\mathcal{E}_{c}$, then $(f, \pi)$ is a non-trivial Walrasian expectations quasi-equilibrium of $\mathcal{E}_{c}$ for some non-zero $\pi: \Omega \rightarrow Y_{+}^{*}$.

Proof. Consider a correspondence $G: I \rightrightarrows\left(Y_{+}\right)^{\Omega}$ defined by $G(t)=\{g(t, \cdot) \in$ $\left.L_{t}: V_{t}(g(t, \cdot))>V_{t}(f(t, \cdot))\right\}$. By $\left(\mathrm{A}_{2}\right), G(t) \neq \emptyset$ for all $t \in I$. Applying the infinite dimensional extension of Lyapunov convexity theorem [25] to the proof of Proposition 5 in [16, p. 62], one can show that

$$
H=\|\cdot\|^{\Omega}-\mathrm{cl}\left(\bigcup\left\{\int_{A} G(t, \cdot) d \mu(t)-\int_{A} a(t, \cdot) d \mu(t): A \in \mathcal{A}, \mu(A)>0\right\}\right)
$$

is a convex subset of $Y^{\Omega}$, where $\mathcal{A}$ denotes the set of Lebesgue measurable subsets of $I$. Since $H \cap \operatorname{int}\left(-Y_{+}\right)^{\Omega}=\emptyset$, by the separation theorem, there is a non-zero positive element $\pi \in\left(Y^{*}\right)^{\Omega}$ such that for any coalition $A$,

$$
\sum_{\omega \in \Omega}\langle\pi(\omega), y(\omega)\rangle \geq \sum_{\omega \in \Omega}\left\langle\pi(\omega), \int_{A} a(t, \omega) d \mu(t)\right\rangle
$$

for all $y \in \int_{A} G(t, \cdot) d \mu(t)$. Let $N_{1}=\left\{i \in N: \sum_{\omega \in \Omega}\left\langle\pi(\omega), a_{i}(\omega)\right\rangle \neq 0\right\}$. Suppose $V_{i}\left(y_{i}\right)>V_{i}\left(x_{i}\right)$ for some $i \in N_{1}$ and $y_{i} \in L_{i}$. If $y_{i} \in B_{i}(\pi)$, by $\left(\mathrm{A}_{1}\right)$, one can construct some $z_{i} \in B_{i}(\pi)$ such that $V_{i}\left(z_{i}\right)>V_{i}\left(x_{i}\right)$ and

$$
\sum_{\omega \in \Omega}\left\langle\pi(\omega), \int_{I_{i}} z_{i}(\omega) d \mu(t)\right\rangle<\sum_{\omega \in \Omega}\left\langle\pi(\omega), \int_{I_{i}} a_{i}(\omega) d \mu(t)\right\rangle
$$


which is a contradiction. Thus, $\sum_{\omega \in \Omega}\left\langle\pi(\omega), y_{i}(\omega)\right\rangle>\sum_{\omega \in \Omega}\left\langle\pi(\omega), a_{i}(\omega)\right\rangle$. By $\left(\mathrm{A}_{2}\right)$, $\sum_{\omega \in \Omega}\left\langle\pi(\omega), x_{i}(\omega)\right\rangle \geq \sum_{\omega \in \Omega}\left\langle\pi(\omega), a_{i}(\omega)\right\rangle$ for all $i \in N_{1}$. Using the feasibility of $f$, one can show that $x_{i} \in B_{i}(\pi)$ for all $i \in N_{1}$. Thus, $(x, \pi)$ is a non-trivial Walrasian expectations quasi-equilibrium in $\mathcal{E}$. By Proposition 2.3 , $(f, \pi)$ is a non-trivial Walrasian expectations quasi-equilibrium in $\mathcal{E}_{c}$.

Corollary 3.2. Let $f$ be a feasible allocation such that $f(t, \cdot)=x_{i}$ for all $t \in I_{i}$ and $i \in N$. Under $\left(\mathrm{A}_{1}\right),\left(\mathrm{A}_{2}\right),\left(\mathrm{A}_{4}\right)$ and $\left(\mathrm{A}_{7}\right), f$ is a Walrasian expectations allocation if and only if $f$ is in the private core of $\mathcal{E}_{c}$.

Next, we extend Theorem 3.1 to an asymmetric information economy with equal treatment property whose commodity space is a Banach lattice containing a quasiinterior point in its positive cone. For each $i \in N$ and each $x_{i} \in L_{i}$, let $P_{i}\left(x_{i}\right):=$ $\left\{y_{i} \in L_{i}: V_{i}\left(y_{i}\right)>V_{i}\left(x_{i}\right)\right\}$ be the set of all $\mathcal{F}_{i}$-measurable consumption bundles preferred to $x_{i}$ by agent $i$. Then $P_{i}: L_{i} \rightrightarrows L_{i}$ is called the preference relation of agent $i$. Note that under $\left(\mathrm{A}_{1}\right)$ and $\left(\mathrm{A}_{3}\right), P_{i}\left(x_{i}\right)$ is convex and relatively $\|\cdot\|^{\Omega}$-open in $L_{i}$ for all $i \in N$. The following definition of ATY-properness is taken from [20].

Definition 3.3. The relation $P_{i}: L_{i} \rightrightarrows L_{i}$ is called $A T Y$-proper at $x_{i} \in L_{i}$ if there exists a convex subset $\widetilde{P}_{i}\left(x_{i}\right)$ of $Y^{\Omega}$ with non-empty $\|\cdot\|^{\Omega}$-interior such that $\widetilde{P}_{i}\left(x_{i}\right) \cap L_{i}=P_{i}\left(x_{i}\right)$ and $\left(\|\cdot\|^{\Omega}-\operatorname{int} \widetilde{P}_{i}\left(x_{i}\right)\right) \cap L_{i} \neq \emptyset$.

$\left(\mathrm{A}_{8}\right)$ Properness. If $\left(x_{1}, \ldots, x_{n}\right)$ is a privately Pareto optimal allocation in $\mathcal{E}$, then for each $i \in N, P_{i}$ is ATY-proper at $x_{i}$.

Lemma 3.4. Let $Y$ be a real vector space endowed with a Hausdorff, locally convex topology $\tau$ and let $U, V$ be convex subsets of $Y$ such that $U$ is open and $U \cap V \neq \emptyset$. Let $y \in V \cap \mathrm{cl} U$, where $\mathrm{cl} U$ denotes the closure of $U$. Suppose that $\pi$ is a linear functional (not necessarily continuous) on $Y$ with $\langle\pi, y\rangle \leq\left\langle\pi, y^{\prime}\right\rangle$ for all $y^{\prime} \in U \cap V$. Then, there exist linear functionals $\pi_{1}$ and $\pi_{2}$ on $Y$ such that $\pi_{1}$ is continuous, $\left\langle\pi_{1}, y\right\rangle \leq\left\langle\pi_{1}, u\right\rangle$ for all $u \in U,\left\langle\pi_{2}, y\right\rangle \leq\left\langle\pi_{2}, v\right\rangle$ for all $v \in V$ and $\pi=\pi_{1}+\pi_{2}$.

Lemma 3.5. Let $Y$ be a Riesz space endowed with a Hausdorff, locally convex topology $\tau$. If $L(z)$ is $\tau$-dense in $Y$, then $L(z)_{+}$is $\tau$-dense in $Y_{+}$.

Lemma 3.6. Let $Y$ be a Riesz space and let $Z$ be an ideal in $Y$. Let $y_{1}, \ldots, y_{m}$ be elements of $Y$ and $z_{1}, \ldots, z_{m}$ be elements of $Z$ such that $\sum_{i=1}^{m} z_{i} \leq \sum_{i=1}^{m} y_{i}$. Suppose that there exists an element $z \in Z$ such that $z \leq y_{i}$ for each $i=1, \ldots, m$. Then, there are elements $\hat{z}_{1}, \ldots, \hat{z}_{m}$ of $Z$ such that $\sum_{i=1}^{m} \hat{z}_{i}=\sum_{i=1}^{m} z_{i}$ and $\hat{z}_{i} \leq y_{i}$ for each $i=1, \ldots, m$.

For proofs of Lemmas 3.4 3.5 and 3.6. see Lemmas 2 and 3 in [18] and Lemma 7 in [20, respectively. In the proof of the next theorem, the argument to get continuity of the equilibrium price is similar to that in Theorem 2 of 20. Our proof needs some additional construction because of the free disposal assumption.

Theorem 3.7. Assume that $\mathcal{E}$ satisfies $\left(\mathrm{A}_{1}\right)-\left(\mathrm{A}_{4}\right),\left(\mathrm{A}_{6}\right)$ and $\left(\mathrm{A}_{8}\right)$. Let $f$ be a feasible allocation in $\mathcal{E}_{c}$ such that $f(t, \cdot)=x_{i}$ for all $t \in I_{i}$ and $i \in N$. If $f$ is in the private core of $\mathcal{E}_{c}$, then $(f, \pi)$ is a non-trivial Walrasian expectations quasiequilibrium of $\mathcal{E}_{c}$ for some non-zero $\pi: \Omega \rightarrow Y_{+}^{*}$.

Proof. Let $f$ be in the private core of $\mathcal{E}_{c}$. Let $Z=L(\hat{a})$, where $\hat{a}$ is selected according to $\left(\mathrm{A}_{6}\right)$. Then, $\left(Z,\|\cdot\|_{\hat{a}}\right)$ is an $A M$-space with $\hat{a}$ as an order unit. Note that $\hat{a} \in\|\cdot\|_{\hat{a}}$-int $Z_{+}, Z_{+}$is $\|\cdot\|_{\hat{a}^{-}}$-closed in $Z$, and the $\|\cdot\|_{\hat{a}^{-}}$-closed unit ball of $Z$ 
coincides with the order interval $[-\hat{a}, \hat{a}]$. Define a new economy $\hat{\mathcal{E}}$ which is identical with $\mathcal{E}$ except for the commodity space being $Z$ equipped with the $\|\cdot\|_{\hat{a}}$-topology, each agent's consumption set being $Z_{+}$in each state of nature $\omega \in \Omega$, and agent $i$ 's ex ante expected utility being $\left.V_{i}\right|_{Z^{\Omega}}$. If $\left(y_{1}, \ldots, y_{n}\right)$ is a feasible allocation of $\mathcal{E}$, then $y_{i}(\omega) \in Z_{+}$for each $i \in N$ and $\omega \in \Omega$. Thus, $x_{i}(\omega) \in Z_{+}$for each $i \in N$ and $\omega \in \Omega$. Since $\sum_{i \in N} a_{i}(\omega)$ is an order unit of $Z, \sum_{i \in N} a_{i}(\omega) \in\|\cdot\|_{\hat{a}}-$ int $Z_{+}$for each $\omega \in \Omega$. Since $\left(Z,\|\cdot\|_{\hat{a}}\right)$ is a Banach lattice, the $\|\cdot\|$-topology is weaker than the $\|\cdot\|_{\hat{a}}$-topology on $Z$. It follows that $\left.U_{i}(\omega, \cdot)\right|_{Z}$ is $\|\cdot\|_{\hat{a}}$-continuous. Thus, we have verified that $\hat{\mathcal{E}}$ satisfies $\left(\mathrm{A}_{1}\right),\left(\mathrm{A}_{2}\right)$ and $\left(\mathrm{A}_{4}\right)$, and $f$ is in the private core of $\hat{\mathcal{E}}_{c}$. By Theorem 3.1] there is a non-zero positive element $\hat{\pi} \in\left(\left(Z,\|\cdot\|_{\hat{a}}\right)^{*}\right)^{\Omega}$ such that $(f, \hat{\pi})$ is a non-trivial Walrasian expectations quasi-equilibrium in $\hat{\mathcal{E}}_{c}$. We need to show that there is a non-zero positive $\pi \in\left((Z,\|\cdot\|)^{*}\right)^{\Omega}$ such that $(f, \pi)$ is a non-trivial Walrasian expectations quasi-equilibrium in $\left.\mathcal{E}_{c}\right|_{Z}$, where $\left.\mathcal{E}_{c}\right|_{Z}$ is identical with $\hat{\mathcal{E}}_{c}$ except for the commodity space being $Z$ with the norm $\|\cdot\|$.

Since $(f, \hat{\pi})$ is a non-trivial Walrasian expectations quasi-equilibrium in $\hat{\mathcal{E}}_{c}$, by Proposition 2.3. $\left(x_{1}, \ldots, x_{n}, \hat{\pi}\right)$ is a non-trivial Walrasian expectations quasi-equilibrium in $\hat{\mathcal{E}}$. Thus, $\left(x_{1}, \ldots, x_{n}\right)$ is a privately Pareto optimal allocation in $\hat{\mathcal{E}}$, and also in $\mathcal{E}$. Let $i \in N$. By $\left(\mathrm{A}_{8}\right)$, there is a convex and $\|\cdot\|^{\Omega}$-open subset $W_{i}$ of $Y^{\Omega}$ such that $\emptyset \neq W_{i} \cap L_{i} \subseteq P_{i}\left(x_{i}\right)$ and $\|\cdot\|^{\Omega}-\operatorname{cl} P_{i}\left(x_{i}\right) \subseteq\|\cdot\|^{\Omega}$-cl $W_{i}$. Since $\sum_{i \in N} a_{i}(\omega)$ is a quasi-interior point of $Y_{+}$for each $\omega \in \Omega, Z$ is $\|\cdot\|$-dense in $Y$. By Lemma 3.5. $Z_{+}$is $\|\cdot\|$-dense in $Y_{+}$. By definition, $L_{i} \cap Z_{+}^{\Omega}$ is $\|\cdot\|^{\Omega}$-dense in $L_{i}$. Thus $W_{i} \cap L_{i} \cap Z_{+}^{\Omega} \neq \emptyset$. Let $Q_{i}=W_{i} \cap Z^{\Omega}$ and $\hat{L}_{i}=L_{i} \cap Z^{\Omega}$. Then, $Q_{i}$ is convex and relatively $\|\cdot\|^{\Omega}$-open in $Z^{\Omega}$. Further, $\emptyset \neq Q_{i} \cap \hat{L}_{i} \subseteq \hat{P}_{i}\left(x_{i}\right)$ and $\|\cdot\|_{Z}^{\Omega}$-cl $\hat{P}_{i}\left(x_{i}\right) \subseteq\|\cdot\|_{Z^{-}}^{\Omega} \operatorname{cl} Q_{i}$, where $\hat{P}_{i}\left(x_{i}\right)=P_{i}\left(x_{i}\right) \cap Z^{\Omega}$. By $\left(\mathrm{A}_{2}\right), x_{i} \in\|\cdot\|_{Z}^{\Omega}$-cl $\hat{P}_{i}\left(x_{i}\right)$, and so $x_{i} \in\|\cdot\|_{Z}^{\Omega}$-cl $Q_{i}$. For any $y_{i} \in Q_{i} \cap \hat{L}_{i}$, since $V_{i}\left(y_{i}\right)>V_{i}\left(x_{i}\right)$ and $\left(x_{1}, \ldots, x_{n}, \hat{\pi}\right)$ is non-trivial Walrasian expectations quasi-equilibrium, $\sum_{\omega \in \Omega}\left\langle\hat{\pi}(\omega), y_{i}(\omega)\right\rangle \geq \sum_{\omega \in \Omega}\left\langle\hat{\pi}(\omega), x_{i}(\omega)\right\rangle$. Since $\Omega$ is finite, $\hat{\pi} \in\left(\left(Z,\|\cdot\|_{\hat{a}}\right)^{\Omega}\right)^{*}$. By Lemma 3.4 there exist a $\pi_{1}^{i} \in\left((Z,\|\cdot\|)^{\Omega}\right)^{*}$ and a linear functional $\pi_{2}^{i}$ on $(Z,\|\cdot\|)^{\Omega}$ such that $\sum_{\omega \in \Omega}\left\langle\pi_{1}^{i}(\omega), x_{i}(\omega)\right\rangle \leq \sum_{\omega \in \Omega}\left\langle\pi_{1}^{i}(\omega), y_{i}(\omega)\right\rangle$ for all $y_{i} \in Q_{i}, \sum_{\omega \in \Omega}\left\langle\pi_{2}^{i}(\omega), x_{i}(\omega)\right\rangle \leq \sum_{\omega \in \Omega}\left\langle\pi_{2}^{i}(\omega), y_{i}(\omega)\right\rangle$ for all $y_{i} \in \hat{L}_{i}$, and $\hat{\pi}=\pi_{1}^{i}+\pi_{2}^{i}$. Since $\hat{L}_{i}$ is a cone, $\sum_{\omega \in \Omega}\left\langle\pi_{2}^{i}(\omega), x_{i}(\omega)\right\rangle=0$. It follows that $\sum_{\omega \in \Omega}\left\langle\pi_{2}^{i}(\omega), y_{i}(\omega)\right\rangle \geq 0$ for all $y_{i} \in \hat{L}_{i}$. Hence, we have

(3.1) $\sum_{\omega \in \Omega}\left\langle\hat{\pi}(\omega), x_{i}(\omega)\right\rangle=\sum_{\omega \in \Omega}\left\langle\pi_{1}^{i}(\omega), x_{i}(\omega)\right\rangle$,

(3.2) $\sum_{\omega \in \Omega}\left\langle\hat{\pi}(\omega), y_{i}(\omega)\right\rangle \geq \sum_{\omega \in \Omega}\left\langle\pi_{1}^{i}(\omega), y_{i}(\omega)\right\rangle$ for all $y_{i} \in \hat{L}_{i}$.

Since $\left(Z,\|\cdot\|_{\hat{a}}\right)$ is a Banach lattice, $\left(Z,\|\cdot\|_{\hat{a}}\right)^{*}$ agrees with the order dual of $Z$. In what follows, let $\left(Z,\|\cdot\|_{\hat{a}}\right)^{*}$ be endowed with the dual ordering relative to the ordering of $Z$. Since each $y_{i} \in \hat{L}_{i}$ can be written as $y_{i}=\sum_{S \in \mathcal{F}_{i}} y_{i}^{S} \mathbf{1}_{S}$, where $y_{i}^{S} \in Z_{+}$and $S \in \mathcal{F}_{i}$, from (3.1) and (3.2), it can be verified that the following hold for all $S \in \mathcal{F}_{i}$ :

(3.3) $\sum_{\omega \in S} \pi_{1}^{i}(\omega) \leq \sum_{\omega \in S} \hat{\pi}(\omega)$,

(3.4) $\sum_{\omega \in S}\left\langle\hat{\pi}(\omega), x_{i}(\omega)\right\rangle=\sum_{\omega \in S}\left\langle\pi_{1}^{i}(\omega), x_{i}(\omega)\right\rangle$.

Since $(Z,\|\cdot\|)$ is a locally solid Riesz space, $(Z,\|\cdot\|)^{*}$ is an ideal in $\left(Z,\|\cdot\|_{\hat{a}}\right)^{*}$. Pick an arbitrary element $S \in \mathcal{F}_{i}$. Since $\hat{\pi}(\omega) \geq 0$ for all $\omega \in S$, by (3.3) and Lemma 3.6. there is an element $\tilde{\pi}^{i} \in\left((Z,\|\cdot\|)^{*}\right)^{S}$ such that $\tilde{\pi}^{i} \leq \hat{\pi}$ on $S$ and $\sum_{\omega \in S} \tilde{\pi}^{i}(\omega)=$ $\sum_{\omega \in S} \pi_{1}^{i}(\omega)$. We claim that for each $\omega \in S,\left\langle\tilde{\pi}^{i}(\omega), x_{i}(\omega)\right\rangle=\left\langle\hat{\pi}(\omega), x_{i}(\omega)\right\rangle$. To 
show this, let $x_{i}=\sum_{R \in \mathcal{F}_{i}} x_{i}^{R} \mathbf{1}_{R}$, where $x_{i}^{R} \in Z_{+}$. By (3.4),

$$
\sum_{\omega \in S}\left\langle\tilde{\pi}^{i}(\omega), x_{i}(\omega)\right\rangle=\left\langle\sum_{\omega \in S} \pi_{1}^{i}(\omega), x_{i}^{S}\right\rangle=\sum_{\omega \in S}\left\langle\hat{\pi}(\omega), x_{i}(\omega)\right\rangle .
$$

Moreover, $\left\langle\tilde{\pi}^{i}(\omega), x_{i}(\omega)\right\rangle \leq\left\langle\hat{\pi}(\omega), x_{i}(\omega)\right\rangle$ for each $\omega \in S$. So, we must have $\left\langle\tilde{\pi}^{i}(\omega), x_{i}(\omega)\right\rangle=\left\langle\hat{\pi}(\omega), x_{i}(\omega)\right\rangle$ for each $\omega \in S$, and the claim is verified. Since $\mathcal{F}_{i}$ is a partition of $\Omega$, there is an element $\tilde{\pi}^{i} \in\left((Z,\|\cdot\|)^{*}\right)^{\Omega}$ such that $\tilde{\pi}^{i} \leq \hat{\pi}$ on $\Omega$ and $\left\langle\tilde{\pi}^{i}(\omega), x_{i}(\omega)\right\rangle=\left\langle\hat{\pi}(\omega), x_{i}(\omega)\right\rangle$ for each $\omega \in \Omega$. Let $N_{0}=N \cup\{0\}$, $\tilde{\pi}^{0}(\omega)=0$ and $a_{0}(\omega)=0$ for each $\omega \in \Omega$. Since $(Z,\|\cdot\|)^{*}$ is an ideal, for each $\omega \in \Omega$, we can choose an element $\ddot{\pi}(\omega) \in(Z,\|\cdot\|)^{*}$ such that $\ddot{\pi}(\omega)=\sup \left\{\tilde{\pi}^{i}(\omega)\right.$ : $\left.i \in N_{0}\right\}$. Then $\ddot{\pi} \in\left((Z,\|\cdot\|)^{*}\right)^{\Omega}$, and $\ddot{\pi} \leq \hat{\pi}$. Define $x_{0} \in Z_{+}^{\Omega}$ such that $x_{0}(\omega)=\sum_{i \in N} a_{i}(\omega)-\sum_{i \in N} x_{i}(\omega)$ for each $\omega \in \Omega$. By the Riesz-Kantorovich formulas, we obtain

$$
\begin{aligned}
\left\langle\ddot{\pi}(\omega), \sum_{i \in N} a_{i}(\omega)\right\rangle & =\sup \left\{\sum_{i \in N_{0}}\left\langle\tilde{\pi}^{i}(\omega), y_{i}\right\rangle: y_{i} \in Z_{+}, \sum_{i \in N_{0}} y_{i}=\sum_{i \in N} a_{i}(\omega)\right\} \\
& \geq \sum_{i \in N_{0}}\left\langle\tilde{\pi}^{i}(\omega), x_{i}(\omega)\right\rangle=\sum_{i \in N}\left\langle\tilde{\pi}^{i}(\omega), x_{i}(\omega)\right\rangle \\
& =\left\langle\hat{\pi}(\omega), \sum_{i \in N} x_{i}(\omega)\right\rangle=\left\langle\hat{\pi}(\omega), \sum_{i \in N} a_{i}(\omega)\right\rangle .
\end{aligned}
$$

Applying $\ddot{\pi} \leq \hat{\pi}$, we have $\left\langle\ddot{\pi}(\omega), \sum_{i \in N} a_{i}(\omega)\right\rangle=\left\langle\hat{\pi}(\omega), \sum_{i \in N} a_{i}(\omega)\right\rangle$ for each $\omega \in \Omega$. Note that $Z=L\left(\sum_{i \in N} a_{i}(\omega)\right)$ for each $\omega \in \Omega$. Let $z \in Z_{+}$be fixed. Choose $\delta>0$ be such that $z \leq \delta \sum_{i \in N} a_{i}(\omega)$ for each $\omega \in \Omega$. Then, $\left\langle\ddot{\pi}(\omega),\left(\delta \sum_{i \in N} a_{i}(\omega)-\right.\right.$ $z)\rangle \leq\left\langle\hat{\pi}(\omega),\left(\delta \sum_{i \in N} a_{i}(\omega)-z\right)\right\rangle$, and so $\langle\ddot{\pi}(\omega), z\rangle \geq\langle\hat{\pi}(\omega), z\rangle$ for each $\omega \in \Omega$. Consequently, $\ddot{\pi} \geq \hat{\pi}$ and therefore, $\ddot{\pi}=\hat{\pi}$. Thus, $\hat{\pi} \in\left((Z,\|\cdot\|)^{*}\right)^{\Omega}$ and $(f, \hat{\pi})$ is a non-trivial Walrasian expectations quasi-equilibrium in $\left.\mathcal{E}_{c}\right|_{Z}$. By the HahnBanach theorem, we can choose a positive element $\pi \in\left((Y,\|\cdot\|)^{*}\right)^{\Omega}$ such that $\pi$ is an extension of $\hat{\pi}$. Since $L_{t} \cap Z_{+}^{\Omega}$ is $\|\cdot\|^{\Omega}$-dense in $L_{t}$ and $V_{t}$ is $\|\cdot\|^{\Omega}$-continuous for each $t \in I$, we deduce that $\sum_{\omega \in \Omega}\langle\pi(\omega), y(\omega)\rangle \geq \sum_{\omega \in \Omega}\langle\pi(\omega), a(t, \omega)\rangle$ for all $y \in L_{t}$ satisfying $V_{t}(y)>V_{t}(f(t, \cdot))$. Further, if $\sum_{\omega \in \Omega}\langle\pi(\omega), a(t, \omega)\rangle>0$, then $\|\cdot\|^{\Omega}$-continuity of $V_{t}$ implies that $(f, \pi)$ is a non-trivial Walrasian expectations quasi-equilibrium of $\mathcal{E}_{c}$. This completes the proof.

Corollary 3.8. Let $f$ be a feasible allocation such that $f(t, \cdot)=x_{i}$ for all $t \in I_{i}$ and $i \in N$. Under $\left(\mathrm{A}_{1}\right)-\left(\mathrm{A}_{4}\right)$ and $\left(\mathrm{A}_{6}\right)-\left(\mathrm{A}_{8}\right), f$ is a Walrasian expectations allocation if and only if $f$ is in the private core of $\mathcal{E}_{c}$.

Now, we extend Theorem 3.7 to an asymmetric information economy with equal treatment property whose commodity space is a Banach lattice having no quasiinterior point in its positive cone. The following definition of strong ATY-properness and the argument to get continuity of the equilibrium price in the next theorem are similar to those in $\left(\mathrm{A} 8^{\prime}\right)$ and Theorem 3 of $[20$.

Definition 3.9. The relation $P_{i}: L_{i} \rightrightarrows L_{i}$ is called strongly ATY-proper at $x_{i} \in L_{i}$ if there is a convex subset $\widehat{P}_{i}\left(x_{i}\right)$ of $Y^{\Omega}$ with non-empty $\|\cdot\|^{\Omega}$-interior such that $\widehat{P}_{i}\left(x_{i}\right) \cap L_{i}=P_{i}\left(x_{i}\right)$ and $\left(\|\cdot\|^{\Omega}-\operatorname{int} \widehat{P}_{i}\left(x_{i}\right)\right) \cap L_{i} \cap L\left(\sum_{i \in N} a_{i}\right) \neq \emptyset$. 
( $\left.\mathrm{A}_{9}\right)$ Strong properness. If $\left(x_{1}, \ldots, x_{n}\right)$ is a privately Pareto optimal allocation in $\mathcal{E}$, then $P_{i}$ is strongly ATY-proper at $x_{i}$ for each $i \in N$.

Theorem 3.10. Assume that $\mathcal{E}$ satisfies $\left(\mathrm{A}_{1}\right)-\left(\mathrm{A}_{3}\right),\left(\mathrm{A}_{4}^{\prime}\right),\left(\mathrm{A}_{6}\right)$ and $\left(\mathrm{A}_{9}\right)$. Let $f$ be a feasible allocation in $\mathcal{E}_{c}$ such that $f(t, \cdot)=x_{i}$ for all $t \in I_{i}$ and $i \in N$. If $f$ is in the private core of $\mathcal{E}_{c}$, then $(f, \pi)$ is a non-trivial Walrasian expectations quasi-equilibrium of $\mathcal{E}_{c}$ for some non-zero $\pi: \Omega \rightarrow Y_{+}^{*}$.

Proof. Let $f$ be in the private core of $\mathcal{E}_{c}$. Let $Z=L(\hat{a})$, where $\hat{a}$ is selected according to $\left(\mathrm{A}_{6}\right)$. Then, $(X,\|\cdot\|)$ equipped with the ordering of $(Y,\|\cdot\|)$ is a Banach lattice, where $X$ denotes the $\|\cdot\|$-closure of $Z$ in $Y$. Note that for any feasible allocation $\left(y_{1}, \ldots, y_{n}\right)$ of $\mathcal{E}, y_{i}(\omega)$ belongs to $Z_{+}$for each $i \in N$ and $\omega \in \Omega$. In particular, $x_{i}(\omega) \in Z_{+}$for each $i \in N$ and $\omega \in \Omega$. Clearly, for each $i \in N$ and $\omega \in \Omega,\left.U_{i}(\omega, \cdot)\right|_{X}$ satisfies $\left(\mathrm{A}_{1}\right)-\left(\mathrm{A}_{3}\right)$. Suppose that $\left(y_{1}, \ldots, y_{n}\right)$ is a privately Pareto optimal allocation in the economy $\left.\mathcal{E}\right|_{X}$, which is identical with $\mathcal{E}$ except for the commodity space being $X$, each agent's consumption set being $X_{+}$in each state of nature $\omega \in \Omega$, and agent $i$ 's ex ante expected utility being $\left.V_{i}\right|_{X^{\Omega}}$. Then $\left(y_{1}, \ldots, y_{n}\right)$ is privately Pareto optimal in $\mathcal{E}$. Take $\widetilde{P}_{i}\left(x_{i}\right)=\widehat{P}_{i}\left(x_{i}\right) \cap X^{\Omega}$ for each $i \in N$, where $\widehat{P}_{i}\left(x_{i}\right)$ is chosen according to $\left(\mathrm{A}_{9}\right)$. So, for each $i \in N, \widetilde{P}_{i}\left(x_{i}\right)$ is convex with nonempty relative $\|\cdot\|^{\Omega}$-interior in $X^{\Omega}$. Let $\hat{L}_{i}=L_{i} \cap X^{\Omega}$ for each $i \in N$. By $\left(\mathrm{A}_{9}\right)$, for each $i \in N, \widetilde{P}_{i}\left(x_{i}\right) \cap \hat{L}_{i}=\left.P_{i}\left(x_{i}\right)\right|_{X^{\Omega}}$, where $\left.P_{i}\left(x_{i}\right)\right|_{X^{\Omega}}$ is the restriction of $P_{i}\left(x_{i}\right)$ to $X^{\Omega}$ and $\left(\operatorname{int} \widetilde{P}_{i}\left(x_{i}\right)\right) \cap \hat{L}_{i} \neq \emptyset$. Thus, $\left(\mathrm{A}_{1}\right)-\left(\mathrm{A}_{4}\right),\left(\mathrm{A}_{6}\right)$ and $\left(\mathrm{A}_{8}\right)$ are satisfied for the economy $\left.\mathcal{E}\right|_{X}$. Note that $f$ is in the private core of $\left.\mathcal{E}_{c}\right|_{X}$. By Theorem 3.7. there exists a non-zero positive element $\pi \in\left(X^{*}\right)^{\Omega}$ such that $(f, \pi)$ is a nontrivial Walrasian expectations quasi-equilibrium in $\left.\mathcal{E}_{c}\right|_{X}$. Therefore, by Proposition 2.3. $\left(x_{1}, \ldots, x_{n}, \pi\right)$ is a non-trivial Walrasian expectations quasi-equilibrium in $\left.\mathcal{E}\right|_{X}$. By the Hahn-Banach theorem, there is a non-zero positive element $\hat{\pi} \in\left(Y^{*}\right)^{\Omega}$ which is an extension of $\pi$. Then $\left(x_{1}, \ldots, x_{n}, \hat{\pi}\right)$ satisfies all conditions of nontrivial Walrasian expectations quasi-equilibrium of $\mathcal{E}$ except for the fact that if $\sum_{\omega \in \Omega}\left\langle\hat{\pi}(\omega), a_{i}(\omega)\right\rangle \neq 0$, then $\sum_{\omega \in \Omega}\left\langle\hat{\pi}(\omega), y_{i}(\omega)\right\rangle>\sum_{\omega \in \Omega}\left\langle\hat{\pi}(\omega), a_{i}(\omega)\right\rangle$ for all $y_{i} \in$ $L_{i}$ satisfying $V_{i}\left(y_{i}\right)>V_{i}\left(x_{i}\right)$. Clearly, this is true for all $y_{i} \in \hat{L}_{i}$.

Since $\left(x_{1}, \ldots, x_{n}, \pi\right)$ is a non-trivial Walrasian expectations quasi-equilibrium in $\left.\mathcal{E}\right|_{X},\left(x_{1}, \ldots, x_{n}\right)$ is privately Pareto optimal in $\left.\mathcal{E}\right|_{X}$ and hence, in $\mathcal{E}$. Pick an $i \in N$. By $\left(\mathrm{A}_{9}\right)$, there is a convex and $\|\cdot\|^{\Omega}$-open subset $Q_{i}$ of $Y^{\Omega}$ such that $\emptyset \neq\left. Q_{i} \cap \hat{L}_{i} \subseteq P_{i}\left(x_{i}\right)\right|_{X^{\Omega}}$ and $\|\cdot\|^{\Omega_{-}}-\operatorname{cl} P_{i}\left(x_{i}\right) \subseteq\|\cdot\|^{\Omega_{-}}-\operatorname{cl} Q_{i}$. By $\left(\mathrm{A}_{2}\right), x_{i} \in\|\cdot\|^{\Omega_{-}}$ $\operatorname{cl} P_{i}\left(x_{i}\right)$ and hence, $x_{i} \in\|\cdot\|^{\Omega_{-}} \operatorname{cl} Q_{i}$. For any $y_{i} \in Q_{i} \cap \hat{L}_{i}$, since $V_{i}\left(y_{i}\right)>V_{i}\left(x_{i}\right)$ and $\left(x_{1}, \ldots, x_{n}, \hat{\pi}\right)$ is a non-trivial Walrasian expectations quasi-equilibrium in $\left.\mathcal{E}\right|_{X}$, $\sum_{\omega \in \Omega}\left\langle\hat{\pi}(\omega), y_{i}(\omega)\right\rangle \geq \sum_{\omega \in \Omega}\left\langle\hat{\pi}(\omega), x_{i}(\omega)\right\rangle$. Note that $\hat{L}_{i}$ is convex, $x_{i} \in \hat{L}_{i}$ and $\hat{\pi} \in$ $\left(Y^{\Omega}\right)^{*}$. By an argument similar to that in Theorem 3.7, we can find an element $\pi_{1}^{i} \in$ $\left(Y^{\Omega}\right)^{*}$ such that $\sum_{\omega \in \Omega}\left\langle\pi_{1}^{i}(\omega), x_{i}(\omega)\right\rangle=\sum_{\omega \in \Omega}\left\langle\hat{\pi}(\omega), x_{i}(\omega)\right\rangle, \sum_{\omega \in \Omega}\left\langle\pi_{1}^{i}(\omega), x_{i}(\omega)\right\rangle \leq$ $\sum_{\omega \in \Omega}\left\langle\pi_{1}^{i}(\omega), y_{i}(\omega)\right\rangle$ for all $y_{i} \in Q_{i}$, and $\sum_{\omega \in \Omega}\left\langle\pi_{1}^{i}(\omega), y_{i}(\omega)\right\rangle \leq \sum_{\omega \in \Omega}\left\langle\hat{\pi}(\omega), y_{i}(\omega)\right\rangle$ for all $y_{i} \in \hat{L}_{i}$. Since $\pi_{1}^{i}$ is $\|\cdot\|^{\Omega_{-}}$-continuous and $\|\cdot\|^{\Omega}$-cl $P_{i}\left(x_{i}\right) \subseteq\|\cdot\|^{\Omega_{-}}$-cl $Q_{i}$, $\sum_{\omega \in \Omega}\left\langle\pi_{1}^{i}(\omega), x_{i}(\omega)\right\rangle \leq \sum_{\omega \in \Omega}\left\langle\pi_{1}^{i}(\omega), y_{i}(\omega)\right\rangle$ for all $y_{i} \in P_{i}\left(x_{i}\right)$. Now, consider elements $\pi_{\star}^{i}, \pi^{\star} \in\left(Y^{\Omega}\right)_{+}^{*}$ defined by $\left\langle\pi_{\star}^{i}, y_{i}\right\rangle=\sum_{\omega \in \Omega}\left\langle\pi_{1}^{i}(\omega), y_{i}^{S}(\omega)\right\rangle$ and $\left\langle\pi^{\star}, y_{i}\right\rangle=$ $\sum_{\omega \in \Omega}\left\langle\hat{\pi}(\omega), y_{i}^{S}(\omega)\right\rangle$, where $y_{i}^{S}=\frac{1}{|S|} \sum_{\omega \in S} y_{i}(\omega)$ for $S \in \mathcal{F}_{i}$. Then $\tilde{\pi}^{i}=\pi_{\star}^{i}+\hat{\pi}-$ $\pi^{\star} \in\left(Y^{\Omega}\right)^{*}$, and it can be verified that

(3.5) $\sum_{\omega \in \Omega}\left\langle\tilde{\pi}^{i}(\omega), x_{i}(\omega)\right\rangle=\sum_{\omega \in \Omega}\left\langle\hat{\pi}(\omega), x_{i}(\omega)\right\rangle$,

(3.6) $\sum_{\omega \in \Omega}\left\langle\tilde{\pi}^{i}(\omega), x_{i}(\omega)\right\rangle \leq \sum_{\omega \in \Omega}\left\langle\tilde{\pi}^{i}(\omega), y_{i}(\omega)\right\rangle$ for all $y_{i} \in P_{i}\left(x_{i}\right)$, and 
(3.7) $\sum_{\omega \in \Omega}\left\langle\tilde{\pi}^{i}(\omega), z(\omega)\right\rangle \leq \sum_{\omega \in \Omega}\langle\hat{\pi}(\omega), z(\omega)\rangle$ for all $z \in X_{+}^{\Omega}$.

Since $Y$ is a locally solid Riesz space, $Y^{*}$ is an ideal in the order dual of $Y$. Let $N_{0}=N \cup\{0\}, \tilde{\pi}^{0}(\omega)=0$ and $a_{0}(\omega)=0$ for each $\omega \in \Omega$. Define $\ddot{\pi} \in$ $\left(Y^{*}\right)^{\Omega}$ such that $\ddot{\pi}(\omega)=\sup \left\{\tilde{\pi}^{i}(\omega): i \in N_{0}\right\}$ for each $\omega \in \Omega$, and $x_{0} \in Z_{+}^{\Omega}$ such that $x_{0}(\omega)=\sum_{i \in N} a_{i}(\omega)-\sum_{i \in N} x_{i}(\omega)$ for each $\omega \in \Omega$. By the RieszKantorovich formulas and techniques similar to those in Theorem 3.7, we have $\left\langle\ddot{\pi}(\omega), \sum_{i \in N} a_{i}(\omega)\right\rangle \geq \sum_{i \in N}\left\langle\tilde{\pi}^{i}(\omega), x_{i}(\omega)\right\rangle$ for each $\omega \in \Omega$. Using (3.5), we can obtain $\sum_{\omega \in \Omega}\left\langle\ddot{\pi}(\omega), \sum_{i \in N} a_{i}(\omega)\right\rangle \geq \sum_{\omega \in \Omega}\left\langle\hat{\pi}(\omega), \sum_{i \in N} a_{i}(\omega)\right\rangle$. Moreover, the RieszKantorovich formulas and (3.7) imply $\sum_{\omega \in \Omega}\langle\ddot{\pi}(\omega), z(\omega)\rangle \leq \sum_{\omega \in \Omega}\langle\hat{\pi}(\omega), z(\omega)\rangle$ for all $z \in X_{+}^{\Omega}$. Since $Z^{\Omega}=L\left(\sum_{i \in N} a_{i}\right), \ddot{\pi} \equiv \hat{\pi}$ on $Z^{\Omega}$, which can be combined with (3.5) and (3.6) to derive $\sum_{\omega \in \Omega}\left\langle\ddot{\pi}(\omega), x_{i}(\omega)\right\rangle \leq \sum_{\omega \in \Omega}\left\langle\ddot{\pi}(\omega), y_{i}(\omega)\right\rangle$ for all $y_{i} \in$ $P_{i}\left(x_{i}\right)$. It follows from $\left(\mathrm{A}_{2}\right)$ and the fact that $\left(x_{1}, \ldots, x_{n}, \ddot{\pi}\right)$ is a non-trivial Walrasian expectations equilibrium in $\left.\mathcal{E}\right|_{X}, \sum_{\omega \in \Omega}\left\langle\ddot{\pi}(\omega), x_{i}(\omega)\right\rangle=\sum_{\omega \in \Omega}\left\langle\ddot{\pi}(\omega), a_{i}(\omega)\right\rangle$. In case that $\sum_{\omega \in \Omega}\left\langle\pi(\omega), a_{i}(\omega)\right\rangle \neq 0$, by $\left(\mathrm{A}_{1}\right), \sum_{\omega \in \Omega}\left\langle\ddot{\pi}(\omega), a_{i}(\omega)\right\rangle<\sum_{\omega \in \Omega}\left\langle\ddot{\pi}(\omega), y_{i}(\omega)\right\rangle$ for all $y_{i} \in P_{i}\left(x_{i}\right)$. Thus, $\left(x_{1}, \ldots, x_{n}, \ddot{\pi}\right)$ is a non-trivial Walrasian expectations quasi-equilibrium in $\mathcal{E}$. Hence, by Proposition 2.3. $(f, \ddot{\pi})$ is a non-trivial Walrasian expectations quasi-equilibrium in $\mathcal{E}_{c}$.

Corollary 3.11. Let $f$ be a feasible allocation such that $f(t, \cdot)=x_{i}$ for all $t \in$ $I_{i}$ and $i \in N$. Under $\left(\mathrm{A}_{1}\right)-\left(\mathrm{A}_{3}\right),\left(\mathrm{A}_{4}^{\prime}\right),\left(\mathrm{A}_{6}\right)-\left(\mathrm{A}_{7}\right)$ and $\left(\mathrm{A}_{9}\right), f$ is a Walrasian expectations allocation if and only if $f$ is in the private core of $\mathcal{E}_{c}$.

3.2. Blocking non-private core allocations. In this subsection, we give an extension of Vind's theorem to an asymmetric information economy with a continuum of agents having the equal treatment property and whose commodity space is a Banach lattice, using the following lemma instead of any convexity theorem on the commodity space.

Lemma 3.12. Assume that $\mathcal{E}$ satisfies $\left(\mathrm{A}_{1}\right),\left(\mathrm{A}_{3}\right)$ and $\left(\mathrm{A}_{5}\right)$. Let $f$ be an allocation such that $f(t, \cdot)=f_{i}$ for all $t \in I_{i}$ and $i \in N$. If $f$ is privately blocked in $\mathcal{E}_{c}$, then it is privately blocked by a coalition $A$ via a function $g$ such that $g(t, \cdot)=g_{i} \in L_{i}$ if $t \in A \cap I_{i}$ and $i \in N$, and $\int_{A}(a(t, \omega)-g(t, \omega)) d \mu(t) \geq z$ for all $\omega \in \Omega$, where $z>0$.

Proof. Since $f$ is privately blocked in $\mathcal{E}_{c}$, there are a coalition $\hat{A} \subseteq I$ and an $\hat{h}: \hat{A} \times \Omega \rightarrow Y_{+}$such that $\hat{h}(t, \cdot) \in L_{t}$ and $V_{t}(\hat{h}(t, \cdot))>V_{t}(f(t, \cdot))$ for all $t \in \hat{A}$, and $\int_{\hat{A}} \hat{h}(t, \omega) d \mu(t) \leq \int_{\hat{A}} a(t, \omega) d \mu(t)$ for all $\omega \in \Omega$. Let $\hat{A}_{i}=\hat{A} \cap I_{i}$ for each $i \in N$, $\hat{N}=\left\{i \in N: \mu\left(\hat{A}_{i}\right) \neq 0\right\}$, and $A=\bigcup_{i \in \hat{N}} \hat{A}_{i}$. For each $i \in \hat{N}$ and $\omega \in \Omega$, put $h_{i}(\omega)=\frac{1}{\mu\left(\hat{A}_{i}\right)} \int_{\hat{A}_{i}} \hat{h}(t, \omega) d \mu(t)$. Then $h_{i} \in L_{i}$ for all $i \in \hat{N}$. Define $h: A \times \Omega \rightarrow Y_{+}$by $h(t, \omega)=h_{i}(\omega)$ if $t \in \hat{A}_{i}$. Clearly, for each $\omega \in \Omega, \int_{A} h(t, \omega) d \mu(t) \leq \int_{A} a(t, \omega) d \mu(t)$, equivalently, $\sum_{i \in \hat{N}} h_{i}(\omega) \mu\left(\hat{A}_{i}\right) \leq \sum_{i \in \hat{N}} a_{i}(\omega) \mu\left(\hat{A}_{i}\right)$. Moreover, concavity of $V_{i}$ implies that $V_{i}(h(t, \cdot))>V_{i}(f(t, \cdot))$ for all $t \in \hat{A}_{i}$ and all $i \in \hat{N}$.

Choose a sequence $\left\{c_{m}\right\} \subseteq(0,1)$ with $c_{m} \rightarrow 0$. For each $i \in \hat{N}$ and each integer $m \geq 1$, define a function $g_{i}^{m}: \Omega \rightarrow Y_{+}$by $g_{i}^{m}(\omega)=\left(1-c_{m}\right) h_{i}(\omega)$. Then $g_{i}^{m} \in L_{i}$ for all $i \in \hat{N}$. For each $i \in \hat{N}$, since $h_{i} \in P_{i}\left(f_{i}\right)$ and $P_{i}\left(f_{i}\right)$ is $\|\cdot\|^{\Omega}$-open, then $g_{i}^{m} \in P_{i}\left(f_{i}\right)$ whenever $m$ is sufficiently large. If we choose such an $m$, then $\sum_{i \in \hat{N}} g_{i}^{m}(\omega) \mu\left(\hat{A}_{i}\right) \leq\left(1-c_{m}\right) \sum_{i \in \hat{N}} a_{i}(\omega) \mu\left(\hat{A}_{i}\right)$, and

$$
\sum_{i \in \hat{N}} a_{i}(\omega) \mu\left(\hat{A}_{i}\right)-\sum_{i \in \hat{N}} g_{i}^{m}(\omega) \mu\left(\hat{A}_{i}\right) \geq c_{m} \sum_{i \in \hat{N}} a_{i}(\omega) \mu\left(\hat{A}_{i}\right) .
$$


Let $z=\inf \left\{c_{m} \sum_{i \in \widehat{N}} a_{i}(\omega) \mu\left(\hat{A}_{i}\right): \omega \in \Omega\right\}$. By $\left(\mathrm{A}_{5}\right)$, we have $z>0$. If we define $g: A \times \Omega \rightarrow Y_{+}$such that $g(t, \omega)=g_{i}^{m}(\omega)$ for all $t \in \hat{A}_{i}$, then $\int_{A}(a(t, \omega)-$ $g(t, \omega)) d \mu(t) \geq z$ for all $\omega \in \Omega$ and $g(t, \cdot) \in L_{t}$ for all $t \in A$, which is required by the lemma.

Theorem 3.13. Assume that $\mathcal{E}$ satisfies $\left(\mathrm{A}_{1}\right)-\left(\mathrm{A}_{3}\right)$ and $\left(\mathrm{A}_{5}\right)$. Let $f$ be a feasible allocation in $\mathcal{E}_{c}$ such that $f(t, \cdot)=f_{i}$ for all $t \in I_{i}$ and $i \in N$. If $f$ is not in the private core of $\mathcal{E}_{c}$, then for any $0<\epsilon<1$, there is a coalition $S$ with $\mu(S)=\epsilon$ privately blocking $f$.

Proof. Since $f$ is not in the private core of $\mathcal{E}_{c}$, by Lemma 3.12, there is a coalition $A \subseteq I$ that privately blocks $f$ via a function $g: A \times \Omega \rightarrow Y_{+}$such that $g(t, \cdot)=$ $g_{i} \in L_{i}$, if $t \in \hat{A}_{i}$ and $\int_{A}(a(t, \cdot)-g(t, \cdot)) d \mu(t) \geq z$, where $\hat{A}_{i}=A \cap I_{i}$ for all $i \in N$. Choose a $\delta \in(0,1)$. Since $\mu$ is atomless, there is $E_{i} \subseteq \hat{A}_{i}$ such that $\mu\left(E_{i}\right)=\delta \mu\left(\hat{A}_{i}\right)$. Moreover, for any $t \in \hat{A}_{i}, a(t, \cdot)-g(t, \cdot)=a_{i}-g_{i}$. Hence,

$$
\int_{E_{i}}(a(t, \cdot)-g(t, \cdot)) d \mu(t)=\left(a_{i}-g_{i}\right) \delta \mu\left(\hat{A}_{i}\right)=\delta \int_{\hat{A}_{i}}(a(t, \cdot)-g(t, \cdot)) d \mu(t) .
$$

Take $E=\bigcup_{i \in \hat{N}} E_{i}$. Then, $\int_{E}(a(t, \cdot)-g(t, \cdot)) d \mu(t)=\delta \int_{A}(a(t, \cdot)-g(t, \cdot)) d \mu(t)$, and $\mu(E)=\delta \mu(A)$. Since $\delta \int_{A}(a(t, \cdot)-g(t, \cdot)) d \mu(t)>0$ for any $0<\delta<1$, there is a coalition $E \subseteq A$ with $\mu(E)=\delta \mu(A)$ privately blocking $f$ via $g$. This proves the theorem for $\epsilon \leq \mu(A)$.

If $\mu(A)=1$, the proof has been completed. Otherwise, $\mu(I \backslash A)>0$, and for any given $0<\epsilon<1$, we define a function $g_{\epsilon}: A \times \Omega \rightarrow Y_{+}$such that $g_{\epsilon}(t, \omega)=$ $\epsilon g(t, \epsilon)+(1-\epsilon) f(t, \omega)$. Then, $g_{\epsilon}(t, \cdot) \in L_{t}$ for all $t \in A$. By concavity of $V_{t}$, we have $V_{t}\left(g_{\epsilon}(t, \cdot)\right)>V_{t}(f(t, \cdot))$ for all $t \in A$. Let $\hat{C}=I \backslash A, C_{i}=\hat{C} \cap I_{i}$, $\hat{N}_{1}=\left\{i \in N: \mu\left(C_{i}\right)>0\right\}$ and $C=\bigcup_{i \in \hat{N}_{1}} C_{i}$. Then $\mu(\hat{C})=\mu(C)$. If $i \in \hat{N}_{1}$, since $\mu$ is atomless, there is $B_{i} \subseteq C_{i}$ such that $\mu\left(B_{i}\right)=(1-\epsilon) \mu\left(C_{i}\right)$. Moreover, $a(t, \cdot)-f(t, \cdot)=a_{i}-f_{i}$ for any $t \in C_{i}$. Hence

$$
\int_{B_{i}}(a(t, \cdot)-f(t, \cdot)) d \mu(t)=(1-\epsilon) \int_{C_{i}}(a(t, \cdot)-f(t, \cdot)) d \mu(t) .
$$

Let $B=\bigcup_{i \in \hat{N}_{1}} B_{i}$. Then $\mu(B)=(1-\epsilon) \mu(I \backslash A)$ and

$$
\int_{B}(a(t, \cdot)-f(t, \cdot)) d \mu(t)=(1-\epsilon) \int_{I \backslash A}(a(t, \cdot)-f(t, \cdot)) d \mu(t) .
$$

Define a consumption bundle $h_{\epsilon}: B \times \Omega \rightarrow Y_{+}$by $h_{\epsilon}(t, \omega)=f(t, \omega)+\frac{\epsilon \mu(A)}{\mu(B)} z$. Since $f(t, \cdot) \in L_{t}$ and $z$ is constant, $h_{\epsilon}(t, \cdot) \in L_{t}$ for all $t \in B$. By monotonicity of $V_{t}$, $V_{t}\left(h_{\epsilon}(t, \cdot)\right)>V_{t}(f(t, \cdot))$ for all $t \in B$.

Let $S=A \cup B$. Then, $\mu(S)=\mu(A)+(1-\epsilon) \mu(I \backslash A)$. Now we show that $S$ privately blocks $f$. Define a consumption bundle $y_{\epsilon}: S \times \Omega \rightarrow Y_{+}$such that $y_{\epsilon}(t, \omega)=g_{\epsilon}(t, \omega)$ if $(t, \omega) \in A \times \Omega$, and $y_{\epsilon}(t, \omega)=h_{\epsilon}(t, \omega)$, if $(t, \omega) \in B \times \Omega$. Clearly, $y_{\epsilon}(t, \cdot) \in L_{t}$ and $V_{t}\left(y_{\epsilon}(t, \cdot)\right)>V_{t}(f(t, \cdot))$ for all $t \in S$. It remains to verify that $y_{\epsilon}$ is feasible for $S$. Since $\int_{A}(a(t, \cdot)-g(t, \cdot)) d \mu(t) \geq z$, we have

$$
\begin{aligned}
\int_{S}\left(a(t, \cdot)-y_{\epsilon}(t, \cdot)\right) d \mu(t) \geq & \epsilon z+(1-\epsilon) \int_{A}(a(t, \cdot)-f(t, \cdot)) d \mu(t) \\
& +\int_{B}(a(t, \cdot)-f(t, \cdot)) d \mu(t)-\epsilon \mu(A) z .
\end{aligned}
$$


On the other hand, $\epsilon z-\epsilon \mu(A) z=\epsilon \mu(I \backslash A) z>0$, and

$$
\int_{B}(a(t, \cdot)-f(t, \cdot)) d \mu(t)=(1-\epsilon) \int_{I \backslash A}(a(t, \cdot)-f(t, \cdot)) d \mu(t) .
$$

Combining the previous few inequalities and equalities, we obtain

$$
\int_{S}\left(a(t, \cdot)-y_{\epsilon}(t, \cdot)\right) d \mu(t)>(1-\epsilon) \int_{I}(a(t, \cdot)-f(t, \cdot)) d \mu(t) \geq 0,
$$

which verifies that $S$ privately blocks $f$ via $y_{\epsilon}$.

\section{Characterizations of Walrasian expectations equilibrium}

In this section, we provide characterizations of Walrasian expectations equilibrium in terms of the private blocking power of the grand coalition. The following concepts for asymmetric information economies in [15] will be needed.

Definition 4.1. A coalition $S \subseteq N$ privately blocks an allocation $x$ of $\mathcal{E}$ in the sense of Aubin via $y=\left(y_{i}\right)_{i \in S}$ if for all $i \in S$, there is $\alpha_{i} \in(0,1]$ such that $V_{i}\left(y_{i}\right)>V_{i}\left(x_{i}\right)$ and $\sum_{i \in S} \alpha_{i} y_{i} \leq \sum_{i \in S} \alpha_{i} a_{i}$. The Aubin private core of $\mathcal{E}$ is the set of all feasible allocations which cannot be blocked by any coalition in the sense of Aubin, and an allocation $x$ in $\mathcal{E}$ is called an Aubin dominated allocation if $x$ is privately blocked by the grand coalition in the sense of Aubin.

The proof of our first theorem in this section is similar to that Theorem 4.2 in [15].

Theorem 4.2. Assume that $\mathcal{E}$ satisfies $\left(\mathrm{A}_{1}\right)-\left(\mathrm{A}_{2}\right),\left(\mathrm{A}_{4}\right)-\left(\mathrm{A}_{5}\right)$ and $\left(\mathrm{A}_{7}\right)$, and its commodity space has an interior point in its positive cone. Then, a feasible allocation $x$ in $\mathcal{E}$ is a Walrasian expectations allocation if and only if $x$ is Aubin non-dominated.

Remark 4.3 ([15]). The participation of an agent $i$ in the grand coalition of $\mathcal{E}$ is said to be close to complete if $\tilde{\alpha}_{i}>1-\delta$ for sufficiently small $\delta>0$. Indeed, Theorem 4.2 shows that the participation of each agent can be chosen to be close to complete. To see this, for any given $0<\delta<1$, by Theorem 3.13, we can choose a privately blocking coalition $S$ such that $\mu(S)>1-\frac{\delta}{n}$. Hence, $\tilde{\alpha}_{i}=n \mu\left(S_{i}\right)>1-\delta$ for all $i \in N$.

Remark 4.4. Conclusions of Theorem 4.2 and Remark 4.3 are also true under $\left(A_{1}\right)-\left(A_{8}\right)$, or $\left.A_{1}\right)-\left(A_{3}\right),\left(A_{5}\right)-\left(A_{7}\right)$ and $\left.\left(A_{9}\right)\right)$, respectively.

The proof of our next theorem is similar to that of Theorem 4.1 in [14, 15]. For an allocation $x=\left(x_{1}, \ldots, x_{n}\right)$ in $\mathcal{E}$ and a vector $r=\left(r_{1}, \ldots, r_{n}\right) \in[0,1]^{n}$, consider an asymmetric information economy $\mathcal{E}(r, x)$ which is identical with $\mathcal{E}$ except for the random initial endowment of each agent $i$ being $a_{i}\left(r_{i}, x_{i}\right)=r_{i} a_{i}+\left(1-r_{i}\right) x_{i}$.

Theorem 4.5. Assume that $\mathcal{E}$ satisfies $\left(\mathrm{A}_{1}\right)-\left(\mathrm{A}_{2}\right),\left(\mathrm{A}_{4}\right)-\left(\mathrm{A}_{5}\right)$ and $\left(\mathrm{A}_{7}\right)$, and its commodity space has an interior point in its positive cone. Then, a feasible allocation $x$ in $\mathcal{E}$ is a Walrasian expectations allocation if and only if $x$ is privately non-dominated in $\mathcal{E}(r, x)$ for any $r \in[0,1]^{n}$.

Remark 4.6 ([15]). Note that for each agent $i \in N, \tilde{r}_{i}$ can be selected arbitrarily close to 1 . To see this, for any given $0<\delta<1$, by Theorem 3.13, we can choose a privately blocking coalition $S$ such that $\mu(S)>1-\frac{\delta}{n}$. Hence, $\tilde{r}_{i}=n \mu\left(S_{i}\right)>1-\delta$ for all $i \in N$. 
Remark 4.7. Conclusion of Theorem4.5 is also true under $\left(A_{1}\right)-\left(A_{8}\right)$, or $\left.A_{1}\right)-\left(A_{3}\right)$, $\left(A_{5}\right)-\left(A_{7}\right)$ and $\left.\left(A_{9}\right)\right)$, respectively. The same is true for Remark 4.6

Remark 4.8 (15]). Theorem 4.5 and the first part of the Remark 4.7not only provide characterizations of Walrasian expectations allocation in terms of veto power of the grand coalition but also implies two welfare theorems as particular cases. If $x$ is a Walrasian expectations allocation in $\mathcal{E}$, then $x$ is a privately Pareto optimal allocation in $\mathcal{E}$ and any $\mathcal{E}(r, x)$ with $r \in[0,1]^{n}$. Thus, the first welfare theorem is an immediate consequence of Theorem 4.5 and the first part of Remark 4.7.

Observe that if $x$ is privately Pareto optimal in $\mathcal{E}$, then $x$ is also privately Pareto optimal in $\mathcal{E}(0, x)$. If we choose $x=a$, then all economies $\mathcal{E}(r, x)$ are equal to $\mathcal{E}(0, x)$ and $x$ is not privately blocked by the grand coalition. If $\inf \left\{x_{i}(\omega): \omega \in \Omega\right\}>0$ for all $i \in N$, Theorem 4.5 and the first part of Remark4.7implies that $x$ is a Walrasian expectations allocation, which is exactly the second welfare theorem.

We conclude this section with some basic examples of Banach lattices and a chart containing a summary where these Banach lattices are applied: (i) $\mathbb{R}^{n}$ : the Euclidean space; (ii) $\ell_{\infty}$ : the space of real bounded sequence with the supremum norm; (iii) $L_{\infty}(\Omega, \Sigma, \mu)$ : the space of essentially bounded, measurable functions on a measure space $(\Omega, \Sigma, \mu)$ with the essential supremum norm; (iv) $C(K)$ : the space of real valued continuous functions on a compact Hausdorff space $K$ with the supremum norm; (v) $\ell_{p}$ : the space of real sequences $x=\left\{x_{n}\right\}$ with the norm $\|x\|_{p}=\left(\sum_{n=1}^{\infty}\left|x_{n}\right|^{p}\right)^{\frac{1}{p}}<\infty$, where $1 \leq p<\infty$; (vi) $L_{p}(\Omega, \Sigma, \mu)$ : the space of measurable functions $f$ on the measure space $(\Omega, \Sigma, \mu)$ with the norm $\|f\|_{p}=$ $\left(\int_{\Omega}|f(\omega)|^{p} d \mu(\omega)\right)^{\frac{1}{p}}<\infty$, where $1 \leq p<\infty$; (vii) $M(K)$ : the space of regular Borel measures on a compact Hausdorff space $K$ with the total variation norm.

\begin{tabular}{|c|c|c|c|}
\hline$Y$ & $\begin{array}{c}Y_{+} \text {having } \\
\text { interior points }\end{array}$ & $\begin{array}{c}Y_{+} \text {having } \\
\text { quasi-interior points }\end{array}$ & Applications \\
\hline $\mathbb{R}^{n}$ & + & + & $\begin{array}{c}\text { Theorems } \\
1,4,5,6\end{array}$ \\
\hline$\ell_{\infty}$ & + & + & $\begin{array}{c}\text { Theorems } \\
1,4,5,6\end{array}$ \\
\hline$L_{\infty}(\Omega, \Sigma, \mu)$ & + & + & $\begin{array}{c}\text { Theorems } \\
1,4,5,6\end{array}$ \\
\hline$C(K)$ & + & + & $\begin{array}{c}\text { Theorems } \\
1,4,5,6\end{array}$ \\
\hline$\ell_{p}$ & + & $\begin{array}{c}\text { Theorems } 2,4 \\
\text { Remarks } 4,6\end{array}$ \\
\hline $\begin{array}{c}L_{p}(\Omega, \Sigma, \mu) \\
(1 \leq p<\infty)\end{array}$ & - & + & $\begin{array}{c}\text { Theorems } 2,4 \\
\text { Remarks } 4,6\end{array}$ \\
\hline $\begin{array}{c}M(K) \\
(K \text { uncountable })\end{array}$ & - & - & $\begin{array}{c}\text { Theorems } 3,4 \\
\text { Remarks } 4,6\end{array}$ \\
\hline
\end{tabular}

\section{Appendix}

(A.1) Results on economies with arbitrary probability spaces of states 
In this section, we apply results established in previous sections, particularly Theorem 3.13 , to characterize the ex-post core and private core of an economy with an arbitrary probability space of states of nature.

Let $\widetilde{\mathcal{E}}$ be a discrete economy identical with $\mathcal{E}$ except for the space of states of nature being a probability measure space $(\Omega, \Sigma, \nu)$, where $\Omega$ is an (infinite) arbitrary set of states of nature, the $\sigma$-algebra $\Sigma$ denotes the set of events and the probability measure $\nu$ denotes the common prior of each agent $i \in N$. For any $x_{i}: \Omega \rightarrow Y_{+}$, the ex ante expected utility of agent $i$ is given by $V_{i}\left(x_{i}\right)=\int_{\Omega} U_{i}\left(\omega, x_{i}(\omega)\right) \nu(d \omega)$. The concepts of an assignment and an allocation in $\widetilde{\mathcal{E}}$ are defined in the same way as that in $\mathcal{E}$. Further, an allocation (assignment) $x$ is said to be feasible if $\sum_{i=1}^{n} x_{i}(\omega) \leq \sum_{i=1}^{n} a_{i}(\omega)$ for almost all $\omega \in \Omega$. A feasible assignment $x$ of $\mathcal{E}$ is called fine non-dominated (resp. weak fine non-dominated) if for all $i \in N, x_{i}$ is $\mathcal{F}_{i}$-measurable (resp. $\bigvee_{i \in N} \mathcal{F}_{i}$-measurable) and there is no feasible assignment $y=\left(y_{1}, \ldots, y_{n}\right)$ such that $y_{i}$ is $\bigvee_{i \in N} \mathcal{F}_{i}$-measurable and $V_{i}\left(y_{i}\right)>V_{i}\left(x_{i}\right)$ for all $i \in N$. A coalition is a non-empty subset $S$ of $N$. A coalition $S \subseteq N$ privately blocks an allocation $x$ in $\widetilde{\mathcal{E}}$ if there exists $y=\left(y_{i}\right)_{i \in S}$ such that $y_{i} \in L_{i}$ and $V_{i}\left(y_{i}\right)>V_{i}\left(x_{i}\right)$ for all $i \in S$, and $\sum_{i \in S} y_{i}(\omega) \leq \sum_{i \in S} a_{i}(\omega)$ for almost all $\omega \in \Omega$. The private core of the economy $\widetilde{\mathcal{E}}$ is the set of all feasible allocations which are not privately blocked by any coalition. A feasible allocation $x$ of $\widetilde{\mathcal{E}}$ is in the ex-post core [8] of $\widetilde{\mathcal{E}}$ if there are no coalition $S \subseteq N, y=\left(y_{i}\right)_{i \in S}$, and $\Omega_{0} \in \Sigma$ such that $\nu\left(\Omega_{0}\right)>0$, $\sum_{i \in S} y_{i}(\omega) \leq \sum_{i \in S} a_{i}(\omega)$ for almost all $\omega \in \Omega_{0}$, and $U_{i}\left(\omega, y_{i}(\omega)\right)>U_{i}\left(\omega, x_{i}(\omega)\right)$ for all $i \in S$ and for almost all $\omega \in \Omega_{0}$.

Assume that $\widetilde{\mathcal{E}}_{c}$ is a continuum economy associated with $\widetilde{\mathcal{E}}$. Since $\mathcal{F}_{t}=\mathcal{F}_{i}$ for each agent $t \in I_{i}$, infinitely many states can be taken in $\widetilde{\mathcal{E}}_{c}$ even agents share their information. An assignment in $\widetilde{\mathcal{E}}_{c}$ is a function $f: I \times \Omega \rightarrow Y_{+}$such that $f(\cdot, \omega) \in L_{1}\left(\mu, Y_{+}\right)$for almost all $\omega \in \Omega$. Let $L_{t}=L_{i}$ for all $t \in I_{i}$ and $i \in N$. An assignment $f$ in $\widetilde{\mathcal{E}}_{c}$ is called an allocation if $f(t, \cdot) \in L_{t}$ for almost all $t \in I$. An allocation $f$ in $\widetilde{\mathcal{E}}_{c}$ is feasible if $\int_{I} f(t, \omega) d \mu(t) \leq \int_{I} a(t, \omega) d \mu(t)$ for almost all $\omega \in \Omega$. A coalition $S$ privately blocks an allocation $f$ in $\widetilde{\mathcal{E}}_{c}$ if there is $g: S \times \Omega \rightarrow Y_{+}$such that $g(t, \cdot) \in L_{t}$ and $V_{t}(g(t, \cdot))>V_{t}(f(t, \cdot))$ for all $t \in S$, and $\int_{S} g(t, \omega) d \mu(t) \leq$ $\int_{S} a(t, \omega) d \mu(t)$ for almost all $\omega \in \Omega$. The private core of $\widetilde{\mathcal{E}}_{c}$ is the set of feasible allocations not privately blocked by any coalition. A feasible allocation $f$ of $\widetilde{\mathcal{E}}_{c}$ is in the ex-post core of $\widetilde{\mathcal{E}}_{c}$ if there are no coalition $S \subseteq I$, function $g: S \times \Omega \rightarrow Y_{+}$, and $\Omega_{0} \in \Sigma$ satisfying $\nu\left(\Omega_{0}\right)>0, \int_{S} g(t, \omega) d \mu(t) \leq \int_{S} a(t, \omega) d \mu(t)$ for almost all $\omega \in \Omega_{0}, U_{t}(\omega, g(t, \omega))>U_{t}(\omega, f(t, \omega))$ for all $t \in S$ and for almost all $\omega \in \Omega_{0}$.

Next, we establish a relation between the set of all fine non-dominated allocations and the ex-post core. This result is new even for finite dimensional commodity space. For an $\omega \in \Omega$, let $\widetilde{\mathcal{E}}_{c}(\omega)$ denote the full information economy whose commodity space being $Y_{+}$, the set of agents being $I$, the utility function and initial endowment of agent $t$ being $U_{t}(\omega, \cdot)$ and $a(t, \omega)$ respectively.

Theorem 4.9. Assume that $\widetilde{\mathcal{E}}$ satisfies $\left(\mathrm{A}_{1}\right)-\left(\mathrm{A}_{3}\right)$ and $\left(\mathrm{A}_{5}\right)$. Let $x$ be a feasible allocation of $\widetilde{\mathcal{E}}$. If $x$ is fine non-dominated in $\widetilde{\mathcal{E}}(r, x)$ for any $r \in[0,1]^{n}$, then $x$ is in the ex-post core of $\widetilde{\mathcal{E}}$.

Proof. Suppose that $x$ is not in the ex-post core of $\widetilde{\mathcal{E}}$. Then, the simple function $f$ on $I$, defined by $f(t, \cdot)=x_{i}$ for all $t \in I_{i}$ and $i \in N$, is not in the ex-post core of $\widetilde{\mathcal{E}}_{c}$. Thus, there is $\Omega_{0} \in \Sigma$ with $\nu\left(\Omega_{0}\right)>0$ such that $f(\cdot, \omega)$ is feasible and not in the 
core of $\widetilde{\mathcal{E}}_{c}(\omega)$ for almost all $\omega \in \Omega_{0}$. Let $A \in \bigvee_{i \in N} \mathcal{F}_{i}$ with $\nu\left(\Omega_{0} \cap A\right) \neq 0$. Applying Theorem 3.13 for each $\widetilde{\mathcal{E}}_{c}(\omega)$ and the fact that $f(t, \cdot)$ and $a(t, \cdot)$ are constant on $\Omega_{0} \cap A$ for all $t \in I$, there exist a coalition $S^{A} \subseteq I$ with $\mu\left(S^{A}\right)>1-\frac{1}{n}$, and a function $g^{A}: S^{A} \times\left(\Omega_{0} \cap A\right) \rightarrow Y_{+}$such that

(A.1.1) $g^{A}(t, \cdot) \in L_{t}$ for all $t \in S^{A}$ and $\int_{S^{A}} g^{A}(t, \omega) d \mu(t) \leq \int_{S^{A}} a(t, \omega) d \mu(t)$ for almost all $\omega \in \Omega_{0} \cap A$,

(A.1.2) $U_{t}\left(\omega, g^{A}(t, \omega)\right)>U_{t}(\omega, f(t, \omega))$ for all $t \in S^{A}$ and for almost all $\omega \in \Omega_{0} \cap A$.

Pick an $\omega_{0} \in \Omega_{0} \cap A$ such that both (A.1.1) and (A.1.2) hold. Now, define a function $h^{A}: S^{A} \times A \rightarrow Y_{+}$such that $h^{A}(t, \omega)=g^{A}\left(t, \omega_{0}\right)$ for all $(t, \omega) \in S^{A} \times A$. Then, $h^{A}(t, \cdot)$ is $\bigvee_{i=1}^{n} \mathcal{F}_{i}$-measurable for all $t \in S^{A}$ and $h^{A}(\cdot, \omega)$ is Bochner integrable for all $\omega \in A$. Since $a(t, \cdot) \in L_{i}$ for all $t \in I_{i}$ and $i \in N, a(\cdot, \omega)=a\left(\cdot, \omega^{\prime}\right)$ for all $\omega, \omega^{\prime} \in A$. Hence, $\int_{S^{A}} h^{A}(t, \omega) d \mu(t) \leq \int_{S^{A}} a(t, \omega) d \mu(t)$ for all $\omega \in A$.

Let $S_{i}^{A}=S^{A} \cap I_{i}$ and $r_{i}^{A}=n \mu\left(S_{i}^{A}\right)$ for all $i \in N$. Since $\mu\left(S^{A}\right)>1-\frac{1}{n}$, we have $r_{i}^{A}>0$ for all $i \in N$. Now for all $i \in N, y_{i}^{A}=\frac{1}{\mu\left(S_{i}^{A}\right)} \int_{S_{i}^{A}} h^{A}(t, \cdot) d \mu(t)$ is $\bigvee_{i=1}^{n} \mathcal{F}_{i}$-measurable. By $\left(\mathrm{A}_{3}\right), U_{i}\left(\omega, y_{i}^{A}(\omega)\right)>U_{i}\left(\omega, x_{i}(\omega)\right)$ for all $i \in N$ and $\omega \in A$. Further, following from $\sum_{i \in N} \mu\left(S_{i}^{A}\right) y_{i}^{A} \leq \sum_{i \in N} \mu\left(S_{i}^{A}\right) a_{i}$, we have $\sum_{i \in N} r_{i}^{A} y_{i}^{A} \leq$ $\sum_{i \in N} r_{i}^{A} a_{i}$. If we put $z_{i}^{A}=r_{i}^{A} y_{i}^{A}+\left(1-r_{i}^{A}\right) x_{i}$ for all $i \in N$, then $z_{i}^{A}$ is $\bigvee_{i=1}^{n} \mathcal{F}_{i^{-}}$ measurable and

$$
\sum_{i \in N} z_{i}^{A} \leq \sum_{i \in N}\left\{r_{i}^{A} a_{i}+\left(1-r_{i}^{A}\right) x_{i}\right\}=\sum_{i \in N} a_{i}\left(r_{i}^{A}, x_{i}\right) .
$$

By $\left(\mathrm{A}_{3}\right)$ again, $U_{i}\left(\omega, z_{i}^{A}(\omega)\right)>U_{i}\left(\omega, x_{i}(\omega)\right)$ for all $i \in N$ and $\omega \in A$. Now for each $i \in N$, the assignment $b_{i}: \Omega \rightarrow Y_{+}$defined by $b_{i}(\omega)=z_{i}^{A}$ if $\omega \in A$ and $b_{i}(\omega)=x_{i}$ if $\omega \notin A$, is $\bigvee_{i=1}^{n} \mathcal{F}_{i}$-measurable, and $b=\left(b_{1}, \ldots, b_{n}\right)$ is feasible in $\widetilde{\mathcal{E}}\left(r^{A}, x\right)$, where $r^{A}=\left(r_{1}^{A}, \ldots, r_{n}^{A}\right)$. Since

$$
V_{i}\left(b_{i}\right)>\int_{A} U_{i}\left(\omega, x_{i}(\omega)\right) \nu(d \omega)+\int_{\Omega \backslash A} U_{i}\left(\omega, x_{i}(\omega)\right) \nu(d \omega)=V_{i}\left(x_{i}\right),
$$

$x$ is fine dominated by $b$ in $\widetilde{\mathcal{E}}\left(r^{A}, x\right)$. The proof is completed.

We conclude this section with a characterization of the private core of $\widetilde{\mathcal{E}}$ by the set of all privately non-dominated allocations of $\widetilde{\mathcal{E}}(r, x)$, which is new even for finite dimensional commodity space.

Theorem 4.10. Assume that $\widetilde{\mathcal{E}}$ satisfies $\left(\mathrm{A}_{1}\right)-\left(\mathrm{A}_{3}\right)$ and $\left(\mathrm{A}_{5}\right)$. Then $x$ is in the private core of $\widetilde{\mathcal{E}}$ if and only if $x$ is a privately non-dominated allocation in $\widetilde{\mathcal{E}}(r, x)$ for any $r \in[0,1]^{n}$.

Proof. Let $x$ be in the private core of $\widetilde{\mathcal{E}}$. Suppose that $x$ is privately dominated in $\widetilde{\mathcal{E}}(r, x)$ for some $r=\left(r_{1}, \ldots, r_{n}\right) \in[0,1]^{n}$. Then, there is an allocation $\left(y_{1}, \ldots, y_{n}\right)$ such that $V_{i}\left(y_{i}\right)>V_{i}\left(x_{i}\right)$ for each $i \in N$ and $\sum_{i \in N} y_{i}(\omega) \leq \sum_{i \in N}\left\{r_{i} a_{i}(\omega)+(1-\right.$ $\left.\left.r_{i}\right) x_{i}(\omega)\right\}$ for almost all $\omega \in \Omega$. Since $x$ is feasible, $\sum_{i \in N} y_{i}(\omega) \leq \sum_{i \in N} a_{i}(\omega)$ for almost all $\omega \in \Omega$, a contradiction with the fact that $x$ is in the private core of $\widetilde{\mathcal{E}}$.

Conversely, let $x$ be a privately non-dominated allocation in $\widetilde{\mathcal{E}}(r, x)$ for any $r \in$ $[0,1]^{n}$. Assume that $x$ is not in the private core of $\widetilde{\mathcal{E}}$. Then, the simple function $f$ on $I$ is not in the private core of $\widetilde{\mathcal{E}}_{c}$ Note that the conclusion of Theorem 3.13 is true in $\widetilde{\mathcal{E}}_{c}$ under $\left(\mathrm{A}_{1}\right)-\left(\mathrm{A}_{3}\right)$ and $\left(\mathrm{A}_{5}\right)$. Then, the conclusion can be derived by an argument similar to that in Theorem 4.5 . 
Let $\widetilde{\mathcal{E}}(s)$ be a symmetric information economy which is identical with $\widetilde{\mathcal{E}}$ except for the information for each agent being $\mathcal{F}_{i}^{s}=\bigvee_{i \in N} \mathcal{F}_{i}$.

Corollary 4.11. Assume that $\widetilde{\mathcal{E}}$ satisfies $\left(\mathrm{A}_{1}\right)-\left(\mathrm{A}_{3}\right)$ and $\left(\mathrm{A}_{5}\right)$. Then, a feasible allocation $x$ is weak fine dominated in $\mathcal{E}(r, x)$ for any $r \in[0,1]^{n}$ if only if $x$ is in the private core of $\widetilde{\mathcal{E}}(s)$.

Remark 4.12. In Section 4, we have applied the equivalence theorems and the Vind's type theorem in Section 3 to establish characterizations of Walrasian expectations allocations for an asymmetric economy with finitely many states of nature. The perspective reader may wonder why such characterizations are not discussed in the scenario where infinitely many states of nature are considered. Although Vind's theorem is valid for an economy with an arbitrary probability space of states of nature, these equivalence theorem are only valid for the scenario where finitely many states of nature are considered. So, the techniques employed in Section 4 do not allow us to establish similar results for characterizing Walrasian expectations allocations in the scenario with infinitely many states of nature. However, the difficulty may be overcome by applying new and different techniques, which could be done in the future work.

\section{(A.2) Discussion on ATY-properness}

In this subsection, we discuss about the ATY-properness of utility functions. Let $A_{i}\left(x_{i}\right)=\left\{y_{i} \in L_{i}: U_{i}\left(\omega, y_{i}(\omega)\right)>U_{i}\left(\omega, x_{i}(\omega)\right)\right.$ for each $\left.\omega \in \Omega\right\}$ for all $x_{i} \in L_{i}$. So, $A_{i}\left(x_{i}\right) \subseteq P_{i}\left(x_{i}\right)$. By $\left(\mathrm{A}_{2}\right), x_{i} \in \operatorname{cl} A_{i}\left(x_{i}\right)$. Then, it is clear from the proof of Theorem 3.7 that we used the following fact from $\left(\mathrm{A}_{8}\right)$ :

(A.2.1) If $x$ is a privately Pareto optimal allocation, then there is a convex and $\|\cdot\|^{\Omega_{\text {-open }}}$ subset $W_{i}$ of $Y^{\Omega}$ such that $\emptyset \neq W_{i} \cap L_{i} \subseteq A_{i}\left(x_{i}\right)$ and $\|\cdot\|^{\Omega_{-}}$ $\operatorname{cl} A_{i}\left(x_{i}\right) \subseteq\|\cdot\|^{\Omega}$-cl $W_{i}$ for each $i \in N$.

We now introduce the definition of ATY-properness of a random utility function similar to that of preference relation.

Definition 4.13. For $\omega \in \Omega$, the utility function $U_{i}(\omega, \cdot)$ is called $A T Y$-proper at $x \in Y_{+}$if there exists a convex subset $\widetilde{A}_{i}(\omega, x)$ of $Y$ with non-empty $\|\cdot\|$-interior such that $\widetilde{A}_{i}(\omega, x) \cap Y_{+}=A_{i}(\omega, x)$ and $\left(\operatorname{int} \widetilde{A}_{i}(\omega, x)\right) \cap Y_{+} \neq \emptyset$, where $A_{i}(\omega, x)=$ $\left\{y \in Y_{+}: U_{i}(\omega, y)>U_{i}(\omega, x)\right\}$.

We need the following assumptions of agents' utility functions.

$\left(\mathrm{A}_{8}^{\prime}\right)$ State Properness. If $\left(x_{i}\right)_{i \in N}$ is a privately Pareto optimal allocation in $\mathcal{E}$, then $U_{i}(\omega, \cdot)$ is ATY-proper at $x_{i}(\omega)$ for each $\omega \in \Omega$ and $i \in N$.

$\left(\mathrm{A}_{8}^{\prime \prime}\right)$ Measurability. For each $i \in N$ and $x \in Y_{+}, U_{i}(\cdot, x)$ is $\mathcal{F}_{i}$-measurable.

Replacing $\left(\mathrm{A}_{8}\right)$ with $\left(\mathrm{A}_{8}^{\prime}\right)$ and $\left(\mathrm{A}_{8}^{\prime \prime}\right)$, we can derive (A.2.1) by taking $W_{i}=\prod_{\omega \in \Omega}$ $\operatorname{int} \widetilde{A}_{i}\left(\omega, x_{i}(\omega)\right)$ for each $i \in N$, where $\widetilde{A}_{i}\left(\omega, x_{i}(\omega)\right)=\widetilde{A}_{i}\left(\omega^{\prime}, x_{i}\left(\omega^{\prime}\right)\right)$ if $\omega, \omega^{\prime} \in R \in$ $\mathcal{F}_{i}$. Alternatively, similar to the $v$-properness of the preference relation in [2, one can assume in the definition of ATY-properness of $U_{i}(\omega, \cdot)$ that $x_{i}(\omega)+y_{i}(\omega) \in$ $\operatorname{int} \widetilde{A}_{i}\left(\omega, x_{i}(\omega)\right)$ for some $y_{i} \in L_{i}$ in the place of $\left(\operatorname{int} \widetilde{A}_{i}(\omega, x)\right) \cap Y_{+} \neq \emptyset$ and we obtain (A.2.1) by using $\left(\mathrm{A}_{8}^{\prime}\right)$ only. Analogous assumptions are not sufficient for 
$\left(\mathrm{A}_{9}\right)$, because the preference $P_{i}\left(x_{i}\right)$ coming from expected utility plays an important role. However, $\left(\mathrm{A}_{8}\right)$ and $\left(\mathrm{A}_{9}\right)$ directly follow from the $v$-properness of the preference relation in a full information economy. To see this, consider a correspondence $S_{i}$ : $Y_{+}^{\Omega} \rightrightarrows Y_{+}^{\Omega}$ defined by $S_{i}(x)=\left\{y \in Y_{+}^{\Omega}: V_{i}(y)>V_{i}(x)\right\}$ for $i \in N$. By $\left(\mathrm{A}_{3}\right), S_{i}\left(x_{i}\right)$ is convex. Choose a non-zero element $v_{i} \in L_{i}$. By the $v_{i}$-properness assumption, there is a convex-valued correspondence $\widetilde{S}_{i}: Y_{+}^{\Omega} \rightrightarrows Y^{\Omega}$ such that for each $x \in Y_{+}^{\Omega}$ the following hold: (i) $x+v_{i} \in\|\cdot\|^{\Omega}$-int $\widetilde{S}_{i}(x)$; and (ii) $\widetilde{S}_{i}(x) \cap Y_{+}^{\Omega}=S_{i}(x)$. Note that if $x \in L_{i}$, then we have $x+v_{i} \in\left(\|\cdot\|^{\Omega}\right.$-int $\left.\widetilde{S}_{i}(x)\right) \cap L_{i}$ and $\widetilde{S}_{i}(x) \cap L_{i}=P_{i}(x)$, and so $P_{i}$ is ATY-proper. Applying a similar argument, one can derive the strong ATY-proper preference relation by taking $v_{i} \in L_{i} \cap L\left(\sum_{i \in N} a_{i}\right)$ for all $i \in N$.

\section{(A.3) Mathematical preliminaries}

Given a real vector space $X$, a function $\|\cdot\|: X \rightarrow[0, \infty)$ satisfying (i) $\|x\| \geq 0$ for all $x \in X$ and $\|x\|=0$ if and only if $x=0$; (ii) $\|\alpha x\|=|\alpha|\|x\|$ for all $x \in X$ and $\alpha \in \mathbb{R}$; (iii) $\|x+y\| \leq\|x\|+\|y\|$ for all $x, y \in X$, is called a norm on $X$, and $(X,\|\cdot\|)$ is called a normed space. If $A$ and $Z$ are subsets of a normed space $(X,\|\cdot\|)$ with $A \subseteq Z$, the closure and the interior of $A$ in the relative topology generated by norm on $Z$ are denoted by $\|\cdot\|_{Z}$-cl $A$ and $\|\cdot\|_{Z}$-int $A$ respectively. When $Z=X$, without confusion, we simply write $\|\cdot\|-\operatorname{cl} A$ and $\|\cdot\|-\operatorname{int} A$ instead. In addition, if $A$ is convex with $\|\cdot\|-\operatorname{int} A \neq \emptyset$, then $\|\cdot\|-\operatorname{cl} A=\|\cdot\|-\operatorname{cl}(\|\cdot\|-\operatorname{int} A)$. If $X$ is a real vector space and $\leq$ is a partial order on $X$, then the pair $(X, \leq)$ is called an ordered vector space if for any $x, y, z \in X$ and any positive real number $\alpha, x \geq y$ implies that $\alpha x+z \geq \alpha y+z$. Recall that a Riesz space is an ordered vector space that is also a lattice, that is, every pair of elements $x, y \in X$ has a supremum $x \vee y$ and an infimum $x \wedge y$. For any element $x$ of a Riesz space, $|x|$ stands for the absolute value of $x$ and is expressed in the form $|x|=x^{+}+x^{-}$, where $x^{+}=x \vee 0$ and $x^{-}=(-x) \vee 0$ are positive and negative parts of $x$ respectively. Note that $x=x^{+}-x^{-}$and $x^{+} \wedge x^{-}=0$. An element $x \in X$ is called a positive element of $X$ if $x \geq 0$ and $X_{+}=\{x \in X: x \geq 0\}$. We write $x>0$, if $x \in X_{+}$and $x \neq 0$. For two points $x, y \in Y, x>y$ means $x-y>0$. If $x \leq y$ in $X$, then $[x, y]$ denotes the order interval $\{z \in X: x \leq z \leq y\}$. A subset $A$ of $X$ is called order bounded if $A \subseteq[x, y]$ for some $x, y \in X$. A norm is called a lattice norm if $|x|<|y|$ implies $\|x\| \leq\|y\|$. A normed Riesz space is a Riesz space with a lattice norm. A complete normed Riesz space is called a Banach lattice. A lattice norm on a Riesz space is an $M$-norm if $\|x \vee y\|=\max \{\|x\|,\|y\|\}$ for any $x, y \geq 0$. An $M$-space is a normed Riesz space with an $M$-norm. A norm complete $M$-space is an $A M$-space. A subset $A$ of a Riesz space is called solid if $|x|<|y|$ and $y \in A$ imply $x \in A$. A solid vector subspace of a Riesz space is called an ideal. A pair $(X, \tau)$ is called a locally solid Riesz space if $X$ is a Riesz space and $\tau$ is a linear topology on $X$ such that $\tau$ has a basis at zero consisting of solid neighborhoods.

A linear functional $f: X \rightarrow \mathbb{R}$ on a Riesz space $X$ is called ordered bounded if $f(A)$ is bounded in $\mathbb{R}$ for any order bounded subset $A$ of $X$. Recall that an order dual $\widetilde{X}$ of a Riesz space $X$ is an ordered vector space consisting of all order bounded linear functionals on $X$ under the usual algebraic operations, and the ordering $f \leq g$ if $\langle f, x\rangle \leq\langle g, x\rangle$ for all $x \in X_{+}$. If $(X,\|\cdot\|)$ is a normed space, its norm dual $X^{*}$ is 
the set of continuous linear functionals on $X$ equipped with the norm $\|\cdot\|$ defined by $\|f\|=\sup \{|\langle f, x\rangle|: x \in X,\|x\| \leq 1\}$. If $(X,\|\cdot\|)$ is a locally solid Riesz space, $X^{*}$ is an ideal in $\widetilde{X}$. If $(X,\|\cdot\|)$ is a Banach lattice, then $X^{*}=\widetilde{X}$ and $\|\cdot\|$-topology is the finest locally solid topology on $X$. The order dual $\widetilde{X}$ of any Riesz space $X$ is an order complete Riesz space, and its lattice operations are given by

$$
\langle f \vee g, x\rangle=\sup \left\{\langle f, y\rangle+\langle g, z\rangle: y, z \in X_{+} \text {and } y+z=x\right\}
$$

and

$$
\langle f \wedge g, x\rangle=\inf \left\{\langle f, y\rangle+\langle g, z\rangle: y, z \in X_{+} \text {and } y+z=x\right\}
$$

for all $f, g \in \tilde{X}$, and $x \in X_{+}$. These two equalities are called the Riesz-Kantorovich formulas. The Hahn-Banach Theorem claims that a continuous linear functional defined on a subspace of a normed space can be extended to a continuous linear functional on the entire space, with its norm preserved. In addition, the separation theorem says for any two disjoint (non-empty) convex subsets $A$ and $B$ in a Banach lattice $(X,\|\cdot\|)$, if either $A$ or $B$ has non-empty interior, then there exists a non-zero $f \in X^{*}$ that separates $A$ and $B$.

Let $X$ be a Banach lattice. If $\Omega$ is a finite set, then $X^{\Omega}$ is endowed with pointwise algebraic operations, the pointwise order and the product norm of $X$ is a Banach lattice. If $x \in(X,\|\cdot\|)^{\Omega}$ (i.e., $X^{\Omega}$ equipped with the $\|\cdot\|^{\Omega}$-topology) and $g \in\left((X,\|\cdot\|)^{\Omega}\right)^{*}$, then there is an element $f \in\left((X,\|\cdot\|)^{*}\right)^{\Omega}$ such that $\langle g, x\rangle=\sum_{\omega \in \Omega}\langle f(\omega), x(\omega)\rangle$ and vise-versa. For any $x \in X$, the principal ideal generated by $x$ is defined by $L(x)=\{y \in X:|y| \leq n|x|$ for some $n \in \mathbb{N}\}$. A point $x \in X_{+}$is called an order unit of $X$ if $L(x)=X$, and a quasi-interior point of $X_{+}$if $L(x)$ is norm dense in $X$ (equivalently, $\langle f, x\rangle>0$ for all $f \in X_{+}^{*} \backslash\{\mathbf{0}\}$ ). If $x$ is a quasi-interior point of $X_{+}$, we write $x \gg 0$. An order unit is a quasiinterior point, but the converse is not true in general. Note that $L(x)$ with the norm $\|y\|_{\hat{x}}=\inf \{\lambda>0:|y|<\lambda|x|\}$ is an $A M$-space with $x$ as an order unit.

Let $(\Omega, \Sigma, \mu)$ be a measure space and $X$ a Banach lattice. A function $\phi: \Omega \rightarrow X$ that assumes only a finite number of values, say $x_{1}, \ldots, x_{n}$, is called a simple function if $A_{i}=\phi^{-1}\left(\left\{x_{i}\right\}\right) \in \Sigma$ for each $i$. As usual, the formula $\phi=\sum_{i=1}^{n} x_{i} \mathbf{1}^{A_{i}}$ is the standard representation of $\phi$, where

$$
\mathbf{1}^{A_{i}}(t)= \begin{cases}1, & \text { if } t \in A_{i} ; \\ 0, & \text { otherwise, }\end{cases}
$$

is called the characteristic function of $A_{i}$ on $\Omega$. Moreover, we say that a function $f: \Omega \rightarrow X$ is $\mu$-measurable if there exists a sequence of simple functions $\left\{\phi_{n}: n \geq\right.$ $1\}$ such that $\lim \left\|f(t)-\phi_{n}(t)\right\|=0$ for almost all $t \in \Omega$. A $\mu$-measurable function $f: \Omega \rightarrow X$ is said to be Bochner integrable if there exists a sequence of simple functions $\left\{\phi_{n}: n \geq 1\right\}$ such that the real measurable function $\left\|f(t)-\phi_{n}(t)\right\|$ is Lebesgue integrable for each $n$ and $\lim \int_{\Omega}\left\|f(t)-\phi_{n}(t)\right\| d \mu(t)=0$. In this case, for each $E \in \Sigma$, the Bochner integral of $f$ over $E$ is defined by $\int_{E} f(t) \mu(t)=$ $\lim \int_{E} \phi_{n}(t) d \mu(t)$, where the last limit is in the norm topology on $X$.

\section{REFERENCES}

[1] Aliprantis, C. D., Border, K. C.: Infinite dimensional analysis: A hitchhiker's guide, Third edition, Springer, 2006

[2] Aliprantis, C. D., Tourky, R., Yannelis, N. C.: Core conditions in general equilibrium theory, J Econ Theory 92, 96-121 (2000) 
[3] Arrow, K. J., Debreu, G.: Existence of an equilibrium for a competitive economy, Econometrica 22, 265-290 (1954)

[4] Aubin, J. P.: Mathematical methods of game and economic theory, North-Holland Publishing Co., Amsterdam-New York, 1979

[5] Aumann, R. J.: Markets with a continuum of traders, Econometrica 32, 39-50 (1964)

[6] Debreu, G.: Theory of value: an axiomatic analysis of economic equilibrium, John Wiley \& Sons, New York (1959)

[7] Einy, E., Moreno, D., Shitovitz, B.: On the core of an economy with differential information, J Econ Theory 94, 262-270 (2000)

[8] Einy, E., Moreno, D., Shitovitz, B.: Competitive and core allocations in large economies with differentiated information, Econ Theory 18, 321-332 (2001)

[9] Evren, Ö., Hüsseinov, F.: Theorems on the core of an economy with infinitely many commodities and consumers, J Math Econ 44, 1180-1196 (2008)

[10] Gabriella Graziano, M., Meo, C.: The Aubin private core of differential information economies, Decisions in Economics and Finance, 28(1), 9-31 (2005).

[11] García-Cutrín, J., Hervés-Beloso, C.: A discrete approach to continuum economies, Econ Theory 3, 577-583 (1993)

[12] Grodal, B.: A second remark on the core of an atomless economy, Econometrica 40, 581-583 (1972)

[13] Hervés-Beloso, C., Moreno-García, E., Núñez-Sanz, C, Páscoa, M. R.: Blocking efficiency of small coalitions in Myopic Economics, J Econ Theory 93, 72-86 (2000)

[14] Hervés-Beloso, C., Moreno-García, E., Yannelis, N. C.: An equivalence theorem for differential information economy, J Math Econ 41, 844-856 (2005a)

[15] Hervés-Beloso, C., Moreno-García, E., Yannelis, N. C.: Characterization and incentive compatibility of Walrasian expectations equilibrium in infinite dimensional commodity spaces, Econ Theory 26, 361-381 (2005b)

[16] Hildenbrand, W.: Core and equilibria in large economies, Priceton University Press, 1974

[17] McKenzie, L.W.: On the existence of general equilibrium for a competitive market, Econometrica 27, 54-71 (1959)

[18] Podczeck, K.: Equilibria in vector lattices without ordered preferences or uniform properness, J Math Econ 25, 465-485 (1996)

[19] Podczeck, K.: Core and Walrasian Equilibria when agents' characteristics are extremely dispersed, Econ Theory 22, 699-725 (2003)

[20] Podczeck, K., Yannelis, N. C.: Equilibrium theory with asymmetric information and with infinitely many commodities, J Econ Theory 141, 152-183 (2008)

[21] Radner, R.: Competitive equilibrium under uncertainty, Econometrica 36, 31-58 (1968)

[22] Radner, R.: Equilibrium under uncertainty, pp. 923-1006 in Handbook of Mathematical Economics Vol 2, North Holland, Amsterdam (1982)

[23] Schmeidler, D.: A remark on the core of an atomless economy, Econometrica 40, 579-580 (1972)

[24] Tourky, R., Yannelis, N. C.: Markets with many more agents than commodities: Aumann's "hidden" assumption, J Econ Theory 101, 189-221 (2001)

[25] Uhl, J. J., Jr.: The range of a vector valued measure, Proc. Amer. Math. Soc. 23, 158-163 (1969)

[26] Vind, K.: A third remark on the core of an atomless economy, Econometrica 40, 585-586 (1972)

[27] Yannelis, N. C.: The core of an economy with differential information, Econ Theory 1, 183$197(1991)$

School of Computing and Mathematical Sciences, Auckland University of Technology, Private Bag 92006, Auckland 1142, New Zealand

E-mail address: anuj.bhowmik@aut.ac.nz

School of Computing and Mathematical Sciences, Auckland University of Technology, Private Bag 92006, Auckland 1142, New Zealand

E-mail address: jiling.cao@aut.ac.nz 